\title{
Supersaturation, counting, and randomness in forbidden subposet problems
}

\author{
Dániel Gerbner \\ Alfréd Rényi Institute of Mathematics \\ Hungary \\ gerbner@renyi.hu

\section{Balázs Patkós} \\ Alfréd Rényi Institute of Mathematics \\ Hungary \\ Moscow Institute of Physics and Technology \\ Russia \\ patkos@renyi.hu \\ Dániel T. Nagy \\ Alfréd Rényi Institute of Mathematics \\ Hungary \\ nagydani@renyi.hu \\ Máté Vizer \\ Alfréd Rényi Institute of Mathematics \\ Hungary \\ vizermate@gmail.com
}

Submitted: July 15, 2020; Accepted: Jan 13, 2021; Published: Feb 26, 2021

(C) The authors. Released under the CC BY-ND license (International 4.0).

\begin{abstract}
In the area of forbidden subposet problems we look for the largest possible size $L a(n, P)$ of a family $\mathcal{F} \subseteq 2^{[n]}$ that does not contain a forbidden inclusion pattern described by $P$. The main conjecture of the area states that for any finite poset $P$ there exists an integer $e(P)$ such that $L a(n, P)=(e(P)+o(1))\left(\begin{array}{c}n \\ \lfloor n / 2\rfloor\end{array}\right)$.

In this paper, we formulate three strengthenings of this conjecture and prove them for some specific classes of posets. (The parameters $x(P)$ and $d(P)$ are defined in the paper.)

- For any finite connected poset $P$ and $\varepsilon>0$, there exists $\delta>0$ and an integer $x(P)$ such that for any $n$ large enough, and $\mathcal{F} \subseteq 2^{[n]}$ of size $(e(P)+\varepsilon)\left(\begin{array}{c}n \\ \lfloor n / 2\rfloor\end{array}\right)$, $\mathcal{F}$ contains at least $\delta n^{x(P)}\left(\begin{array}{c}n \\ \lfloor n / 2\rfloor\end{array}\right)$ copies of $P$.

- The number of $P$-free families in $2^{[n]}$ is $2^{(e(P)+o(1))\left(\begin{array}{c}n \\ \lfloor n / 2\rfloor\end{array}\right)}$.

- Let $\mathcal{P}(n, p)$ be the random subfamily of $2^{[n]}$ such that every $F \in 2^{[n]}$ belongs to $\mathcal{P}(n, p)$ with probability $p$ independently of all other subsets $F^{\prime} \in 2^{[n]}$. For any finite poset $P$, there exists a positive rational $d(P)$ such that if $p=\omega\left(n^{-d(P)}\right)$, then the size of the largest $P$-free family in $\mathcal{P}(n, p)$ is $(e(P)+o(1)) p\left(\begin{array}{c}n \\ \lfloor n / 2\rfloor\end{array}\right)$ with high probability.
\end{abstract}

Mathematics Subject Classifications: 05D05 


\section{Introduction}

Extremal set theory starts with the seminal result of Sperner [21] that was generalized by Erdös [7] as follows: if a family $\mathcal{F} \subseteq 2^{[n]}$ of sets does not contain a nested sequence $F_{1} \subsetneq F_{2} \subsetneq \cdots \subsetneq F_{k+1}$ (such nested sequences are called chains of length $k+1$ or $(k+1)$ chains for short), then its size cannot exceed that of the union of $k$ middle levels of $2^{[n]}$, i.e., $|\mathcal{F}| \leqslant \sum_{i=1}^{k}\left(\left\lfloor\frac{n}{\lfloor-k}\right\rfloor+i\right)$. This theorem has many applications and several of its variants have been investigated.

In the early 80's, Katona and Tarján [13] introduced the following general framework to study set families avoiding some fixed inclusion patterns: we say that a subfamily $\mathcal{G}$ of $\mathcal{F}$ is a (non-induced) copy of a poset $(P, \leqslant)$ in $\mathcal{F}$, if there exists a bijection $i: P \rightarrow \mathcal{G}$ such that if $p, q \in P$ with $p \leqslant q$, then $i(p) \subseteq i(q)$. If $i$ satisfies the property that for $p, q \in P$ we have $p \leqslant q$ if and only if $i(p) \subseteq i(q)$, then $\mathcal{G}$ is called an induced copy of $P$ in $\mathcal{F}$. If $\mathcal{F}$ does not contain any (induced) copy of $P$, the $\mathcal{F}$ is said to be (induced) $P$-free. The largest possible size of a(n induced) $P$-free family $\mathcal{F} \subseteq 2^{[n]}$ is denoted by $L a(n, P)\left(L a^{*}(n, P)\right)$. Let $P_{k}$ denote the $k$-chain, then the result of Erdös mentioned above determines $\operatorname{La}\left(n, P_{k+1}\right)$. These parameters have attracted the attention of many researchers, and there are widely believed conjectures in the area (see Conjecture 1 ) that appeared first in [3] and [11], giving the asymptotics of $L a(n, P)$ and $L a^{*}(n, P)$.

Let $e(P)$ denote the maximum integer $m$ such that for any $i \leqslant n$, the family $\left(\begin{array}{c}{[n]} \\ i+1\end{array}\right) \cup$ $\left(\begin{array}{c}{[n]} \\ i+2\end{array}\right) \cup \cdots \cup\left(\begin{array}{c}{[n]} \\ i+m\end{array}\right)$ is $P$-free. Similarly, let $e^{*}(P)$ denote the maximum integer $m$ such that for any $i \leqslant n$, the family $\left(\begin{array}{c}{[n]} \\ i+1\end{array}\right) \cup\left(\begin{array}{c}{[n]} \\ i+2\end{array}\right) \cup \cdots \cup\left(\begin{array}{c}{[n]} \\ i+m\end{array}\right)$ is induced $P$-free.

Conjecture 1. (i) $L a(n, P)=(e(P)+o(1))\left(\begin{array}{c}n \\ \lfloor n / 2\rfloor\end{array}\right)$.

(ii) $L a^{*}(n, P)=\left(e^{*}(P)+o(1)\right)\left(\begin{array}{c}n \\ \lfloor n / 2\rfloor\end{array}\right)$.

Conjecture 1 has been verified for several classes of posets, but is still open in general. For more results on the $L a(n, P)$ function, see Chapter 7 of [8], and see other chapters for more background on the generalizations considered in this paper.

After determining (the asymptotics of) the extremal size and the structure of the extremal families, one may continue in several directions. Stability results state that all $P$-free families having almost extremal size must be very similar in structure to the middle $e(P)$ levels of $2^{[n]}$. Supersaturation problems ask for the minimum number of copies of $P$ that a family $\mathcal{F} \subseteq 2^{[n]}$ of size $L a(n, P)+E$ may contain. This is clearly at least $E$, but usually one can say much more. Counting problems ask to determine the number of $P$-free families in $2^{[n]}$. As any subfamily of a $P$-free family is $P$-free, therefore the number of $P$-free families is at least $2^{\operatorname{La}(n, P)}$. The question is how many more such families there are. Finally, one can address random versions of the forbidden subposet problem. Let $\mathcal{P}(n, p)$ denote the probability space of all subfamilies of $2^{[n]}$ such that for any $F \subseteq[n]$, the probability that $F$ belongs to $\mathcal{P}(n, p)$ is $p$, independently of any other set $F^{\prime}$. What is the size of the largest $P$-free subfamily of $\mathcal{P}(n, p)$ with high probability ${ }^{1}$ ? Clearly, for

\footnotetext{
${ }^{1}$ we say that a sequence of events $E_{1}, E_{2}, \ldots, E_{n}, \ldots$ holds with high probability (or w.h.p., in short) if $\mathbb{P}\left(E_{n}\right)$ tends to 1 as $n$ tends to infinity
} 
$p=1$, this is $L a(n, P)$. For other values of $p$, an obvious construction is to take a $P$-free subfamily of $2^{[n]}$, and then the sets that are in $\mathcal{P}(n, p)$ form a $P$-free family. Taking the $e(P)$ middle levels shows that the size of the largest $P$-free family in $\mathcal{P}(n, P)$ is at least $p(e(P)+o(1))\left(\begin{array}{c}n \\ \lfloor n / 2\rfloor\end{array}\right)$ w.h.p.. For what values of $p$ does this formula give the asymptotically correct answer?

In this paper, we will consider supersaturation, counting and random versions of the forbidden subposet problem, mostly focusing on supersaturation results. We will propose three strengthenings of Conjecture 1 and prove them for some classes of posets. In the remainder of the introduction, we state our results and also what was known before.

The supersaturation version of Sperner's problem is to determine the minimum number of pairs $F \subsetneq F^{\prime}$ over all subfamilies of $2^{[n]}$ of given size. We say that a family $\mathcal{F}$ is centered if it consists of the sets closest to $n / 2$. More precisely, if $F \in \mathcal{F}$ and ||$G|-n / 2|<|| F|-n / 2|$ imply $G \in \mathcal{F}$. Kleitman [14] proved that among families of cardinality $m$, centered ones contain the smallest number of copies of $P_{2}$. He conjectured that the same holds for any $P_{k}$. After several partial results, e.g. $[2,5,6]$, the conjecture was confirmed by Samotij [20]. The following is a consequence of the result of Samotij. We will only use it with $k=2$, i.e. the result of Kleitman.

Theorem 1. For any $k, t$ with $k-1 \leqslant t$ and $\varepsilon>0$ there exists $n_{k, t, \varepsilon}$ such that if $n \geqslant n_{k, t, \varepsilon}$, then any family $\mathcal{F} \subseteq 2^{[n]}$ of size at least $(t+\varepsilon)\left(\begin{array}{c}n \\ \lfloor n / 2\rfloor\end{array}\right)$ contains at least $\varepsilon \frac{n^{t}}{2^{t+1}}\left(\begin{array}{c}n \\ \lfloor n / 2\rfloor\end{array}\right)$ chains of length $k$.

Apart from the above, the only supersaturation result in the area of forbidden subposet problems is due to Patkós [19]. It gives the minimum number of copies of the butterfly poset $^{2} B$ in families of size $L a(n, B)+E$ for small values of $E$.

We will investigate the number of copies of $P$ created when the number of additional sets compared to a largest $P$-free family is proportional to the size of the middle level $\left(\begin{array}{c}{[n]} \\ {[n / 2\rfloor}\end{array}\right)$. Let $M(n, P)$ denote the number of copies of $P$ in the $e(P)+1$ middle levels of $2^{[n]}$, and let $M^{*}(n, P)$ denote the number of induced copies of $P$ in the $e^{*}(P)+1$ middle levels of $2^{[n]}$. The Hasse diagram of a poset $P$ is the directed graph with vertex set $P$ and for $p, q \in P,(p q)$ is an arc in the Hasse diagram if $p<q$ and there does not exist $z \in P$ with $p<z<q$. We say that $P$ is connected, if its Hasse diagram (as a digraph) is weakly connected, i.e., we cannot partition its vertices into two sets such that there is no arc between those sets. The undirected Hasse diagram is the undirected graph obtained from the Hasse diagram by removing orientations of all arcs.

Proposition 2. For any connected poset $P$ on at least two elements there exist positive integers $x(P)$ and $x^{*}(P)$ such that $M(n, P)=\Theta\left(n^{x(P)}\left(\begin{array}{c}n \\ \lfloor n / 2\rfloor\end{array}\right)\right)$ and $M^{*}(n, P)=$ $\Theta\left(n^{x^{*}(P)}\left(\begin{array}{c}n \\ \lfloor n / 2\rfloor\end{array}\right)\right)$ hold.

Proof. The proofs of the two statements are analogous, so we include only that of the non-induced version. For a copy $\mathcal{G}$ of $P$ with all sets from the $e(P)+1$ middle levels of

\footnotetext{
${ }^{2}$ the poset on four elements $a, b<c, d$
} 
$2^{[n]}$, let $A_{\mathcal{G}}=\cap_{G \in \mathcal{G}} G, B_{\mathcal{G}}=\cup_{G \in \mathcal{G}} G$ and $m_{\mathcal{G}}=\left|A_{\mathcal{G}}\right|, M_{\mathcal{G}}=\left|B_{\mathcal{G}}\right|$. Let us define $x(P)$ now. Let $x(P):=\max _{\mathcal{G}}\left\{M_{\mathcal{G}}-m_{\mathcal{G}}\right\}$, where the maximum runs through all the copies $\mathcal{G}$ of $P$ with all sets from the $e(P)+1$ middle levels of $2^{[n]}$.

We claim that for any such $\mathcal{G}$, we have $M_{\mathcal{G}}-m_{\mathcal{G}} \leqslant e(P)|P|$ (in other words $x(P) \leqslant$ $e(P)|P|)$. Indeed, as $P$ is connected, we can go through its elements in an order such that every element is in relation with at least one of the earlier elements. As $\mathcal{G}$ is from the $e(P)+1$ middle levels, this means that the new element is a set that is either contained in, or contains an earlier set, thus it differs from that set in at most $e(P)$ elements. In the first case, this new element decreases $m_{\mathcal{G}}$ by at most $e(P)$, in the second case it increases $M_{\mathcal{G}}$ by at most $e(P)$, so we are done.

Similarly, one can show $\left|m_{\mathcal{G}}-n / 2\right|,\left|M_{\mathcal{G}}-n / 2\right| \leqslant e(P)|P|$. Clearly, for any $A \subseteq B$ with $|B|-|A| \leqslant e(P)|P|$ there is at most a fixed constant number of copies $\mathcal{G}$ of $P$ such that $A=A_{\mathcal{G}}$ and $B=B_{\mathcal{G}}$. Finally, the number of pairs $A \subseteq B$, with $|B \backslash A| \leqslant x(P)$, ||$A|-n / 2| \leqslant e(P)|P|$ and ||$B|-n / 2| \leqslant e(P)|P|$ is at most $\bar{C}\left(\begin{array}{c}n \\ \lfloor n / 2\rfloor\end{array}\right)(\underset{x(P)}{\lfloor n / 2\rfloor+e(P)|P|})$. This yields $M(n, P)=O\left(n^{x(P)}\left(\begin{array}{c}n \\ \lfloor n / 2\rfloor\end{array}\right)\right)$.

For the lower bound, fix a copy $\mathcal{G}$ with $\left|B_{\mathcal{G}} \backslash A_{\mathcal{G}}\right|=x(P)$. Clearly, for any $A^{\prime} \subseteq B^{\prime}$ with $\left|A^{\prime}\right|=\left|A_{\mathcal{G}}\right|,\left|B^{\prime}\right|=\left|B_{\mathcal{G}}\right|$ there exists a permutation $\pi$ of $[n]$ with $\pi\left(A_{\mathcal{G}}\right)=A^{\prime}$ and $\pi\left(B_{\mathcal{G}}\right)=B^{\prime}$. Therefore, such permutations $\pi$ map $\mathcal{G}$ into distinct copies of $P$. Their number is clearly at least $\left(\begin{array}{c}n \\ \left|A_{\mathcal{G}}\right|\end{array}\right)\left(\begin{array}{c}n-\left|A_{\mathcal{G}}\right| \\ \left|B_{\mathcal{G}} \backslash A_{\mathcal{G}}\right|\end{array}\right) \geqslant c \cdot n^{x(P)}\left(\begin{array}{c}n \\ \lfloor n / 2\rfloor\end{array}\right)$ for some positive constant $c$.

Now we can state the first generalization of Conjecture 1.

Conjecture 3. (i) For every poset $P$ and $\varepsilon>0$ there exists $\delta>0$ such that if $\mathcal{F} \subseteq 2^{[n]}$ is of size at least $(e(P)+\varepsilon)\left(\begin{array}{c}n \\ \lfloor n / 2\rfloor\end{array}\right)$, then $\mathcal{F}$ contains at least $\delta \cdot M(n, P)$ many copies of $P$.

(ii) For every poset $P$ and $\varepsilon>0$ there exists $\delta>0$ such that if $\mathcal{F} \subseteq 2^{[n]}$ is of size at least $\left(e^{*}(P)+\varepsilon\right)\left(\begin{array}{c}n \\ \lfloor n / 2\rfloor\end{array}\right)$, then $\mathcal{F}$ contains at least $\delta \cdot M^{*}(n, P)$ many induced copies of $P$.

We will prove Conjecture 3 for several classes of tree posets. A poset $T$ is a tree poset, if its undirected Hasse diagram is a tree. The height $h(P)$ of poset $P$ is the length of the longest chain in $P$. Note that for any tree poset $T$ of height 2 , we have $x(T)=x^{*}(T)=|T|-1$.

Theorem 2. Let $T$ be any height 2 tree poset of $t+1$ elements. Then for any $\varepsilon>0$ there exist $\delta>0$ and $n_{0}$ such that for any $n \geqslant n_{0}$ any family $\mathcal{F} \subseteq 2^{[n]}$ of size $|\mathcal{F}| \geqslant(1+\varepsilon)\left(\begin{array}{c}n \\ \lfloor n / 2\rfloor\end{array}\right)$ contains at least $\delta n^{t}\left(\begin{array}{c}n \\ \lfloor n / 2\rfloor\end{array}\right)$ copies of $T$.

For two elements $x, y$ of the poset $P$, we write $x \prec y$ if $x<_{P} y$ and there does not exist any $z \in P$ with $x<_{P} z<_{P} y$. We say that a tree poset $T$ is upward (downward) monotone, if for any $x \in T$ there exists at most 1 element $y \in T$ with $y \prec x(x \prec y)$. A tree poset is called monotone, if it is either upward or downward monotone. 
Theorem 3. For any monotone tree poset $T$ and $\varepsilon>0$, there exist $\delta>0$ and $n_{0}$ such that for any $n \geqslant n_{0}$ any family $\mathcal{F} \subseteq 2^{[n]}$ of size $|\mathcal{F}| \geqslant(h(T)-1+\varepsilon)\left(\begin{array}{c}n \\ \lfloor n / 2\rfloor\end{array}\right)$ contains at least $\delta n^{x(T)}\left(\begin{array}{c}n \\ \lfloor n / 2\rfloor\end{array}\right)$ copies of $T$.

The complete multipartite poset $K_{r_{1}, r_{2}, \ldots, r_{\ell}}$ is a poset on $\sum_{i=1}^{\ell} r_{i}$ elements $a_{i, j}$ with $i=1,2, \ldots, \ell, j=1,2, \ldots, r_{i}$ such that $a_{i, j}<a_{i^{\prime}, j^{\prime}}$ if and only if $i<i^{\prime}$. The poset $K_{1, r}$ is usually denoted by $\vee_{r}$, and the poset $K_{r, 1}$ is denoted by $\wedge_{r}$. The poset $K_{s, 1, t}$ is a tree poset with $x\left(K_{s, 1, t}\right)=x^{*}\left(K_{s, 1, t}\right)=s+t$.

Theorem 4. For any $s, t \in \mathbb{N}$ and $\varepsilon>0$ there exist $n_{0}=n_{\varepsilon, s, t}$ and $\delta>0$ such that any $\mathcal{F} \subseteq 2^{[n]}$ of size at least $(2+\varepsilon)\left(\begin{array}{c}n \\ \lfloor n / 2\rfloor\end{array}\right)$ with $n \geqslant n_{0}$ contains at least $\delta n^{s+t}\left(\begin{array}{c}n \\ \lfloor n / 2\rfloor\end{array}\right)$ induced copies of $K_{s, 1, t}$.

We will consider the supersaturation problem for the generalized diamond $D_{s}$, i.e., the poset on $s+2$ elements with $a<b_{1}, b_{2}, \ldots, b_{s}<c$. For any integer $s \geqslant 2$, let us define $m_{s}=\left\lceil\log _{2}(s+2)\right\rceil$ and $m_{s}^{*}=\min \left\{m: s \leqslant\left(\begin{array}{c}m \\ \lceil m / 2\rceil\end{array}\right)\right\}$. Clearly, for any integer $s \geqslant 2$, we have $e\left(D_{s}\right)=x\left(D_{s}\right)=m_{s}$ and $e^{*}\left(D_{s}\right)=x^{*}\left(D_{s}\right)=m_{s}^{*}$. The next theorem establishes a lower bound that is less by a factor of $\sqrt{n}$ than what Conjecture 3 states for diamond posets $D_{s}$ for infinitely many $s$.

Theorem 5. (i) If $s \in\left[2^{m_{s}-1}-1,2^{m_{s}}-\left(\begin{array}{c}m_{s} \\ \left\lceil\frac{m_{s}}{2}\right\rceil\end{array}\right)-1\right]$, then for any $\varepsilon>0$ there exists a $\delta>0$ such that every $\mathcal{F} \subseteq 2^{[n]}$ with $|\mathcal{F}| \geqslant\left(m_{s}+\varepsilon\right)\left(\begin{array}{c}n \\ \lfloor n / 2\rfloor\end{array}\right)$ contains at least $\delta \cdot n^{m_{s}-0.5}\left(\begin{array}{c}n \\ \lfloor n / 2\rfloor\end{array}\right)$ copies of $D_{s}$.

(ii) For any $\varepsilon>0$ there exists a $\delta>0$ such that every $\mathcal{F} \subseteq 2^{[n]}$ with $|\mathcal{F}| \geqslant(4+\varepsilon)\left(\begin{array}{c}n \\ \lfloor n / 2\rfloor\end{array}\right)$ contains at least $\delta \cdot n^{3.5}\left(\begin{array}{c}n \\ \lfloor n / 2\rfloor\end{array}\right)$ induced copies of $D_{4}$.

(iii) For any constant $c$ with $1 / 2<c<1$ there exists an integer $s_{c}$ such that if $s \geqslant s_{c}$ and $s \leqslant c\left(\begin{array}{c}m_{s}^{*} \\ \left.m_{s}^{*} / 2\right\rfloor\end{array}\right)$, then the following holds: for any $\varepsilon>0$ there exists a $\delta>0$ such that every $\mathcal{F} \subseteq 2^{[n]}$ with $|\mathcal{F}| \geqslant\left(m_{s}^{*}+\varepsilon\right)\left(\begin{array}{c}n \\ \lfloor n / 2\rfloor\end{array}\right)$ contains at least $\delta \cdot n^{m_{s}^{*}-0.5}\left(\begin{array}{c}n \\ \lfloor n / 2\rfloor\end{array}\right)$ induced copies of $D_{s}$.

Let us elaborate on the statement of Theorem 5. Part (i) partitions the integers according to powers of 2 and states that for every integer $k$, and for most of the integers $s$ in the interval $\left[2^{k}-1,2^{k+1}-2\right]$, the poset $D_{s}$ possesses this weak supersaturation property. By "most of the integers" we mean that the ratio of integers for which the statement holds and the length of the interval tends to 1 as $k$ tends to infinity. The smallest value of $s$ that (i) applies to is $s=3$ with $m_{s}=3$ as then $3 \in\left[2^{3-1}-1,2^{3}-\left(\begin{array}{l}3 \\ 2\end{array}\right)-1\right]=[3,4]$. Part (ii) is about supersaturation of induced copies of $D_{4}$. Part (iii) is similar to (i) but again about induced copies of $D_{s}$. This time positive integers are partitioned into intervals according to the sequence $\left\{\left(\begin{array}{c}k \\ \lfloor k / 2\rfloor\end{array}\right)\right\}_{k=1}^{\infty}$, namely $\left\{\left[\left(\begin{array}{c}k \\ \lfloor k / 2\rfloor\end{array}\right)+1,\left(\begin{array}{c}k+1 \\ \lfloor(k+1) / 2\rfloor\end{array}\right)\right]\right\}_{k=1}^{\infty}$. As $k$ tends to infinity, the ratio of right and left endpoints tends to 2. Part (iii) states that as $k$ tends to infinity, those integers $s$ in the initial segment of the $k$ th interval for which $D_{s}$ has the claimed supersaturation property, take up larger and larger ratio of the interval. 
Let us turn our attention to counting (induced) $P$-free families. As we mentioned earlier, every subfamily of a $P$-free family is $P$-free, therefore $2^{\operatorname{La(n,P)}} \geqslant 2^{(e(P)+o(1))\left(\begin{array}{c}n \\ \lfloor n / 2\rfloor\end{array}\right)}$ is a lower bound on the number of such families. Determining the number of $P_{2}$-free families has attracted a lot of attention. The upper bound $2^{(1+o(1))\left(\begin{array}{c}n \\ \lfloor n / 2\rfloor\end{array}\right)}$, asymptotically matching in the exponent the trivial lower bound was obtained by Kleitman [15]. After several improvements, Korshunov [16] determined asymptotically the number of $P_{2}$-free families.

Conjecture 4. (i) The number of $P$-free families in $2^{[n]}$ is $2^{(e(P)+o(1))\left(\begin{array}{c}n \\ \lfloor n / 2\rfloor\end{array}\right)}$.

(ii) The number of induced $P$-free families in $2^{[n]}$ is $2^{\left(e^{*}(P)+o(1)\right)\left(\begin{array}{c}n \\ \lfloor n / 2\rfloor\end{array}\right)}$.

Theorem 6. (i) The number of induced $\vee_{r+1}$-free families is $2^{(1+o(1))\left(\begin{array}{c}n \\ \lfloor n / 2\rfloor\end{array}\right)}$.

(ii) The number of induced $K_{s, 1, t}$ free families in $2^{[n]}$ is $2^{(2+o(1))\left(\begin{array}{c}n \\ \lfloor n / 2\rfloor\end{array}\right)}$.

As every height 2 poset $P$ is a non-induced subposet of $K_{|P|, 1,|P|}$, Conjecture 4 (i) is an immediate consequence of Theorem 6 for those height 2 posets $P$ for which $e(P)=2$.

Finally, we turn to random versions of forbidden subposet problems. The probabilistic version of Sperner's theorem was proved by Balogh, Mycroft, and Treglown [1] and Collares and Morris [17], independently. It states that if $p=\omega(1 / n)$, then the largest antichain in $\mathcal{P}(n, p)$ is of size $(1+o(1)) p\left(\begin{array}{c}n \\ \lfloor n / 2\rfloor\end{array}\right)$ w.h.p.. This is sharp in the sense that if $p=o(1 / n)$ then the asymptotics is different. Note that as any $k$-Sperner family is the union of $k$ antichains, the analogous statement holds for $k$-Sperner families in $\mathcal{P}(n, p)$. Both papers used the container method. Hogenson in her $\mathrm{PhD}$ thesis [12] adapted the method of Balogh, Mycroft, and Treglown to obtain the same results for non-induced $\vee_{r}$-free families.

Let us state a general proposition that gives a range of $p$ when one can have a $P$-free family in $\mathcal{P}(n, p)$ that is larger than $p(e(P)+o(1))\left(\begin{array}{c}{[n} \\ \lfloor n / 2\rfloor\end{array}\right)$.

Proposition 5. For any finite connected poset $P$, the following statements hold.

(i) If $p=o\left(n^{-\frac{x(P)}{|P|-1}}\right)$ and $p\left(\begin{array}{c}n \\ \lfloor n / 2\rfloor\end{array}\right) \rightarrow \infty$, then the largest $P$-free family in $\mathcal{P}(n, p)$ has size at least $(e(P)+1-o(1)) p\left(\begin{array}{c}n \\ \lfloor n / 2\rfloor\end{array}\right)$ w.h.p..

(ii) If $p=o\left(n^{-\frac{x^{*}(P)}{|P|-1}}\right)$ and $p\left(\begin{array}{c}n \\ \lfloor n / 2\rfloor\end{array}\right) \rightarrow \infty$, then the largest induced $P$-free family in $\mathcal{P}(n, p)$ has size at least $\left(e^{*}(P)+1-o(1)\right) p\left(\begin{array}{c}n \\ \lfloor n / 2\rfloor\end{array}\right)$ w.h.p..

Proof. We only prove $(i)$, the proof of $(i i)$ is similar. Let us denote the random family of the $e(P)+1$ middle levels after keeping any of its sets with probability $p$ by $\mathcal{M}_{p}$. Clearly, $\mathbb{E}\left(\left|\mathcal{M}_{p}\right|\right)=(e(P)+1+o(1)) p\left(\begin{array}{c}n \\ \lfloor n / 2\rfloor\end{array}\right)$ and as and $p\left(\begin{array}{c}n \\ \lfloor n / 2\rfloor\end{array}\right) \rightarrow \infty$, we have $\left|\mathcal{M}_{p}\right|=$ $(e(P)+1+o(1)) p\left(\begin{array}{c}n \\ \lfloor n / 2\rfloor\end{array}\right)$ w.h.p.. Let $X$ be the random variable that denotes the number of copies of $P$ in $\mathcal{M}_{p}$. Then we have $\mathbb{E}(X)=\Theta\left(p^{|P|} n^{x(P)}\left(\begin{array}{c}n \\ \lfloor n / 2\rfloor\end{array}\right)\right)$. By the assumption on $p$, we 
have $p^{|P|} n^{x(P)}\left(\begin{array}{c}n \\ \lfloor n / 2\rfloor\end{array}\right)=o\left(p\left(\begin{array}{c}n \\ \lfloor n / 2\rfloor\end{array}\right)\right)$, and so $X=o\left(\left|\mathcal{M}_{p}\right|\right)$ w.h.p., and thus by removing the copies of $P$ from $\mathcal{M}_{p}$, we obtain a $P$-free family in $\mathcal{P}(n, p)$ of size $(e(P)+1-o(1)) p\left(\begin{array}{c}n \\ \lfloor n / 2\rfloor\end{array}\right)$ w.h.p..

If $\mathcal{M}_{p}$ does not contain a subposet $P^{\prime}$ of $P$, then it is $P$-free, thus we have the following.

Corollary 6. For any finite poset $P$, let $d(P)=\min \frac{x\left(P^{\prime}\right)}{\left|P^{\prime}\right|-1}$, where $P^{\prime}$ runs through all connected subposets $P^{\prime}$ of $P$ with $e(P)=e\left(P^{\prime}\right)$. Similarly, let $d^{*}(P)=\min \frac{x^{*}\left(P^{\prime}\right)}{\left|P^{\prime}\right|-1}$, where $P^{\prime}$ runs through all connected subposets $P^{\prime}$ of $P$ with $e^{*}(P)=e^{*}\left(P^{\prime}\right)$. Then the following statements hold.

(i) If $p=o\left(n^{-d(P)}\right)$, then the largest $P$-free family in $\mathcal{P}(n, p)$ has size at least $(e(P)+$ $1-o(1)) p\left(\begin{array}{c}n \\ \lfloor n / 2\rfloor\end{array}\right)$ w.h.p.

(ii) If $p=o\left(n^{-d^{*}(P)}\right)$, then the largest induced $P$-free family in $\mathcal{P}(n, p)$ has size $\left(e^{*}(P)+\right.$ $1-o(1)) p\left(\begin{array}{c}n \\ \lfloor n / 2\rfloor\end{array}\right)$ w.h.p.

We conjecture that the bounds above are sharp.

Conjecture 7. For any finite connected poset $P$ the following statements hold.

(i) If $p=\omega\left(n^{-d(P)}\right)$, then the largest $P$-free family in $\mathcal{P}(n, p)$ has size $(e(P)+o(1)) p\left(\begin{array}{c}n \\ \lfloor n / 2\rfloor\end{array}\right)$ w.h.p..

(ii) If $p=\omega\left(n^{-d^{*}(P)}\right)$, then the largest induced $P$-free family in $\mathcal{P}(n, p)$ has size $\left(e^{*}(P)+\right.$ $o(1)) p\left(\begin{array}{c}n \\ \lfloor n / 2\rfloor\end{array}\right)$ w.h.p..

The following theorem verifies Conjecture 7 for the posets $\vee_{r+1}$ and $K_{s, 1, t}$. Observe that $d\left(\vee_{r+1}\right)=d^{*}\left(\vee_{r+1}\right)=1$ for all $r \geqslant 0$ and $d\left(K_{s, 1, t}\right)=d^{*}\left(K_{s, 1, t}\right)=2$ for all $s, t \geqslant 1$.

Theorem 7. If $p=\omega(1 / n)$, then the following are true.

(i) For any integer $r \geqslant 0$, the largest induced $\vee_{r+1}$-free family in $\mathcal{P}(n, p)$ has size $(1+o(1)) p\left(\begin{array}{c}n \\ \lfloor n / 2\rfloor\end{array}\right)$ w.h.p..

(ii) For any pair $s, t \geqslant 1$ of integers, the largest induced $K_{s, 1, t}$ free family in $\mathcal{P}(n, p)$ has size $(2+o(1)) p\left(\begin{array}{c}n \\ \lfloor n / 2\rfloor\end{array}\right)$ w.h.p..

The structure of the paper is as follows: in the next section, we gather some earlier results that will be used as tools in the proofs of our theorems. Also, we will obtain an induced $\vee_{r+1}$-free container lemma. Section 3, in three subsections, contain the proofs of our theorems. 


\section{Preliminaries}

Here and in the next section, we will assume that $n$ is large enough whenever it is necessary.

Lemma 8. For any $1 \leqslant l \leqslant n / 2$ we have $\sum_{i=0}^{l-1}\left(\begin{array}{c}n \\ i\end{array}\right) \leqslant 2 \sqrt{n}\left(\begin{array}{l}n \\ l\end{array}\right)$.

Proof. Let $m=\lfloor\sqrt{n}\rfloor$ and observe that for any $k<n / 2$ we have

$$
\frac{\left(\begin{array}{c}
n \\
k-m
\end{array}\right)}{\left(\begin{array}{l}
n \\
k
\end{array}\right)} \leqslant \frac{\left(\begin{array}{c}
n \\
\lfloor n / 2\rfloor-m
\end{array}\right)}{\left(\begin{array}{c}
n \\
\lfloor n / 2\rfloor
\end{array}\right)} \leqslant \prod_{i=1}^{m} \frac{\lfloor n / 2\rfloor-i+1}{\lceil n / 2\rceil+i} \leqslant e^{-\sum_{i=1}^{m} \frac{2 i}{n}} \leqslant e^{-1} .
$$

So dividing $\sum_{i=0}^{l-1}\left(\begin{array}{c}n \\ i\end{array}\right)$ into $m$ subsums depending on the residue of $i \bmod m$, we obtain subsums that can be upper bounded by geometric progressions of quotient $e^{-1}$.

We denote by $\mathbf{C}_{k}$ the set of chains of length $k+1$, i.e., the set of maximal chains in $2^{[k]}$. The next two lemmas could be stated as bounds on the expected value of the size of the intersection of a $D_{s}$-free family $\mathcal{F}$ and a random maximal chain, and a family without antichains of size $s$ a random maximal chain, respectively. We state them in the form that will be most convenient for our purposes.

Lemma 9 (Griggs, Li, Lu, in the proof of Theorem 2.5 in [10]).

If $s \in\left[2^{m_{s}-1}-1,2^{m_{s}}-\left(\begin{array}{c}m_{s} \\ \left\lceil\frac{m_{s}}{2}\right\rceil\end{array}\right)-1\right]$, and $\mathcal{G} \subseteq 2^{[k]}$ is a $D_{s}$-free family of sets, then the number of pairs $(G, \mathcal{C})$ with $G \in \mathcal{G} \cap \mathcal{C}$ and $\mathcal{C} \in \mathbf{C}_{k}$ is at most $m_{s} k$ !.

Lemma 10 (Patkós [18]). (i) Let $\mathcal{G} \subseteq 2^{[k]}$ be a family of sets such that any antichain $\mathcal{A} \subset \mathcal{G}$ has size at most 3. Then the number of pairs $(G, \mathcal{C})$ with $G \in \mathcal{G} \cap \mathcal{C}$ and $\mathcal{C} \in \mathbf{C}_{k}$ is at most $4 k !$.

(ii) For any constant $c$ with $1 / 2<c<1$ there exists an integer $s_{c}$ such that if $s \geqslant s_{c}$ and $s \leqslant c\left(\begin{array}{c}m_{s}^{*} \\ \left\lceil m_{s}^{*} / 2\right\rceil\end{array}\right)$, then the following holds: if $\mathcal{G} \subseteq 2^{[k]}$ is a family of sets such that any antichain $\mathcal{A} \subset \mathcal{G}$ has size less than $s$, then the number of pairs $(G, \mathcal{C})$ with $G \in \mathcal{G} \cap \mathcal{C}$ and $\mathcal{C} \in \mathbf{C}_{k}$ is at most $m_{s}^{*} k !$.

The special case $r=1$ of the next theorem was proved by Carroll and Katona [4].

Theorem 8 (Griggs, Li [9]). If $\mathcal{F} \subseteq 2^{[n]}$ is an induced $\vee_{r+1}$-free family, then $|\mathcal{F}| \leqslant$ $\left(1+\frac{2 r}{n}+O\left(\frac{r}{n^{2}}\right)\right)\left(\begin{array}{c}n \\ \lfloor n / 2\rfloor\end{array}\right)$ holds, where the constant in the $O$-term does not depend on $r$.

We remark that we will use the above theorem with $r=\Theta(n)$.

Theorem 9. For every $\varepsilon>0$ and $t, r \in \mathbb{Z}^{+}$, if $n$ is large enough and $\mathcal{F} \subseteq 2^{[n]}$ is of size at least $(t+\varepsilon)\left(\begin{array}{c}n \\ \lfloor n / 2\rfloor\end{array}\right)$, then there exists an induced copy of $\vee_{\delta n^{t}}$ in $\mathcal{F}$ with $\delta=\frac{\varepsilon}{2^{t+3} t !}$.

Proof. As $\left|\left\{G \subseteq[n]:|| G|-n / 2| \geqslant n^{2 / 3}\right\}\right| \leqslant \frac{1}{n^{2}}\left(\begin{array}{c}n \\ \lfloor n / 2\rfloor\end{array}\right)$, we can assume that $n / 2-n^{2 / 3} \leqslant$ $|F| \leqslant n+n^{2 / 3}$ holds for every $F \in \mathcal{F}$ and $|\mathcal{F}| \geqslant(t+\varepsilon / 2)\left(\begin{array}{c}n \\ \lfloor n / 2\rfloor\end{array}\right)$. The Lubell-function 
$\lambda_{n}(\mathcal{F}):=\sum_{F \in \mathcal{F}} \frac{1}{\left(\begin{array}{c}n \\ |F|\end{array}\right)}$ of a family $\mathcal{F} \subseteq 2^{[n]}$ of sets is the average number of sets in $\mathcal{F} \cap \mathcal{C}$ over all maximal chains $\mathcal{C} \in \mathbf{C}_{n}$. Clearly, $|\mathcal{F}| \geqslant(t+\varepsilon / 2)\left(\begin{array}{c}n \\ \lfloor n / 2\rfloor\end{array}\right)$ implies $\lambda_{n}(\mathcal{F}) \geqslant t+\varepsilon / 2$.

Let $\mathbf{C}_{F}$ be the collection of maximal chains $\mathcal{C} \in \mathbf{C}_{n}$ such that $F$ is the smallest set in $\mathcal{F} \cap \mathcal{C}$, and let $\mathbf{C}_{\emptyset}$ be the collection of those maximal chains that avoid $\mathcal{F}$. Let us partition $\mathbf{C}_{n}$ into $\mathbf{C}_{\emptyset} \cup \bigcup_{F \in \mathcal{F}} \mathbf{C}_{F}$. Writing $\mathcal{F}_{F}=\left\{F^{\prime} \backslash F: F^{\prime} \in \mathcal{F}, F \subseteq F^{\prime}\right\}$, we obtain $\lambda_{n}(\mathcal{F})=\sum_{F \in \mathcal{F}} \frac{\left|\mathbf{C}_{F}\right|}{n !} \lambda_{n-|F|}\left(\mathcal{F}_{F}\right)$. This means that for some $F \in \mathcal{F}$, we must have $\lambda_{n-|F|}\left(\mathcal{F}_{F}\right) \geqslant t+\varepsilon / 2$. If $\mathcal{F}$ does not contain any induced copy of $\vee_{\delta n^{t}}$, then for any $i \geqslant t$ we have $\left|\left\{F^{\prime} \in \mathcal{F}_{F}:\left|F^{\prime}\right|=i\right\}\right|<\delta n^{t}$, thus writing $\mathcal{F}_{F, 1}=\left\{G \in \mathcal{F}_{F}:|G| \leqslant t-1\right\}$ and $\mathcal{F}_{F, 2}=\mathcal{F}_{F} \backslash \mathcal{F}_{F, 1}$ we obtain

$$
\begin{aligned}
& t+\varepsilon / 2 \leqslant \lambda_{n-|F|}\left(\mathcal{F}_{F}\right)=\lambda_{n-|F|}\left(\mathcal{F}_{F, 1}\right)+\lambda_{n-|F|}\left(\mathcal{F}_{F, 2}\right) \leqslant \\
& \leqslant t+\delta n^{t} \sum_{i=t}^{2 n^{2 / 3}} \frac{1}{\left(\begin{array}{c}
n-|F| \\
i
\end{array}\right)}<t+\frac{2 \delta n^{t}}{\left(\begin{array}{c}
n / 2-n^{2 / 3} \\
t
\end{array}\right)} \leqslant t+2^{t+2} \delta t !
\end{aligned}
$$

This is a contradiction if $\delta \leqslant \frac{\varepsilon}{2^{t+3} t !}$.

Hogenson [12], altering a proof of Balogh, Mycroft, and Treglown [1], obtained a container lemma for non-induced $\vee_{r+1}$-free families. With the help of Theorem 9 , we can validate it for induced $\vee_{r+1}$-free families.

For a family $\mathcal{G}$ of sets in $2^{[n]}$ and a set $F \subseteq 2^{[n]}$, we introduce $U_{\mathcal{G}}(F)=\{G \in \mathcal{G}$ : $F \subseteq G\}$. Previous proofs used the $\mathcal{G}$-degree of $F$, the size of $U_{\mathcal{G}}(F)$. Observe that $\mathcal{F}$ is $\vee_{r+1}$-free if and only if the $\mathcal{F}$-degree of every $F \in \mathcal{F}$ is at most $r$. We introduce the $\mathcal{G}$-weight of $F$ as the size of the largest antichain in $U_{\mathcal{G}}(F)$. Observe that $\mathcal{F}$ is induced $\vee_{r+1}$-free if and only if the $\mathcal{F}$-weight of every $F \in \mathcal{F}$ is at most $r$. Now we will state the appropriate container lemma. For the reader not familiar with container lemmas: the aim of the next theorem is to prove the existence of "not many" containers such that every induced $\vee_{r+1}$-free family is contained in one of them. The containers are of the form $\mathcal{H}_{1} \cup \mathcal{H}_{2} \cup g\left(\mathcal{H}_{1} \cup \mathcal{H}_{2}\right)$ and their number is relatively small as they are determined by $\mathcal{H}_{1}$ and $\mathcal{H}_{2}$ and the size of these is much smaller than that of asymptotically maximum sized $\vee_{r+1}$-free families.

Theorem 10. Let $t, r \in \mathbb{Z}^{+}$and $\varepsilon \leqslant \frac{1}{(2 t)^{t+1}}$ and assume $n$ is large enough. Then there exist functions $f:\left(\begin{array}{c}2^{[n]} \\ \leqslant(r+1) 2^{n} n^{-(t+0.9)}\end{array}\right) \rightarrow\left(\begin{array}{c}2^{[n]} \\ (t+1+\varepsilon)\left(\begin{array}{c}n \\ \lfloor n / 2\rfloor\end{array}\right)\end{array}\right)$ and $g:\left(\begin{array}{c}2^{[n]} \\ \leqslant(r+1) \frac{t+2}{\varepsilon^{2} n^{t}}\left(\begin{array}{c}n \\ \lfloor n / 2\rfloor\end{array}\right)\end{array}\right) \rightarrow\left(\begin{array}{c}2^{[n]} \\ (t+\varepsilon)\left(\begin{array}{l}n \\ \lfloor n / 2\rfloor\end{array}\right)\end{array}\right)$ such that for any induced $\vee_{r+1}$-free family $\mathcal{F} \subseteq 2^{[n]}$ there exist disjoint subfamilies $\mathcal{H}_{1}, \mathcal{H}_{2} \subseteq \mathcal{F}$ with $\left|\mathcal{H}_{1}\right| \leqslant(r+1) 2^{n} n^{-(t+0.9)}$ and $\left|\mathcal{H}_{1}\right|+\left|\mathcal{H}_{2}\right| \leqslant(r+1) \frac{t+2}{\varepsilon^{2} n^{t}}\left(\begin{array}{c}n \\ \lfloor n / 2\rfloor\end{array}\right)$ such that $\left(\mathcal{H}_{1} \cup \mathcal{H}_{2}\right) \cap g\left(\mathcal{H}_{1} \cup \mathcal{H}_{2}\right)=\emptyset, \mathcal{H}_{2} \subseteq f\left(\mathcal{H}_{1}\right)$ and $\mathcal{F} \subseteq \mathcal{H}_{1} \cup \mathcal{H}_{2} \cup g\left(\mathcal{H}_{1} \cup \mathcal{H}_{2}\right)$.

Proof. The proof uses the standard graph container algorithm with some modifications. First, we fix an ordering $S_{1}, S_{2}, \ldots, S_{2^{n}}$ of $2^{[n]}$ and also an ordering $\mathcal{S}_{1}, \mathcal{S}_{2}, \ldots, \mathcal{S}_{2^{2^{n}}}$ of $2^{2^{[n]}}$. The input of the algorithm is an induced $\vee_{r+1}$-free family $\mathcal{F} \subseteq 2^{[n]}$, and in two phases it outputs $\mathcal{H}_{1}, \mathcal{H}_{2}, f\left(\mathcal{H}_{1}\right)$ and $g\left(\mathcal{H}_{1} \cup \mathcal{H}_{2}\right)$ as follows.

At the beginning we set $\mathcal{G}^{0}=2^{[n]}, \mathcal{H}_{1}^{0}=\mathcal{H}_{2}^{0}=\emptyset$ and we start at Phase I. 
Later in the $i$ th round (for $i=1,2, \ldots$ ) we always pick a $G_{i} \in \mathcal{G}^{i-1}$ with largest $\mathcal{G}^{i-1}$-weight. If there are several sets $G$ with the same (largest) $\mathcal{G}^{i-1}$-weight, we pick the one appearing first in our fixed ordering of $2^{[n]}$.

\section{Phase I.}

- If $G_{i} \notin \mathcal{F}$, we set $\mathcal{G}^{i}=\mathcal{G}^{i-1} \backslash\{G\}$ and $\mathcal{H}_{1}^{i}=\mathcal{H}_{1}^{i-1}, \mathcal{H}_{2}^{i}=\mathcal{H}_{2}^{i-1}$.

- If $G_{i} \in \mathcal{F}$, and the $\mathcal{G}^{i-1}$-weight of $G_{i}$ is more than $n^{t+0.9}$, then we pick the largest antichain $\mathcal{A}^{i}$ in $U_{\mathcal{G}^{i-1}}\left(G_{i}\right)$. If there are multiple such antichains, we pick the one with the smallest index in our fixed ordering of $2^{2^{[n]}}$ and set $\mathcal{G}^{i}=\mathcal{G}^{i-1} \backslash\left(\mathcal{A}^{i} \cup\left\{G_{i}\right\}\right), \mathcal{H}_{1}^{i}=$ $\mathcal{H}_{1}^{i-1} \cup\left[\left(\mathcal{A}^{i} \cap \mathcal{F}\right) \cup\left\{G_{i}\right\}\right]$ and $\mathcal{H}_{2}^{i}=\mathcal{H}_{2}^{i-1}$.

- If $G_{i} \in \mathcal{F}$, and the $\mathcal{G}^{i-1}$-weight of $G_{i}$ is at most $n^{t+0.9}$, then Phase I is ended, we keep $\mathcal{G}^{i}=\mathcal{G}^{i-1}, \mathcal{H}_{2}^{i}=\mathcal{H}_{2}^{i-1}$, set $\mathcal{H}_{1}=\mathcal{H}_{1}^{i-1}$ (from here on, $\mathcal{H}_{1}^{i}$ does not change) and define $f\left(\mathcal{H}_{1}\right)=\mathcal{G}^{i}$. And jump to Phase II.

Phase II.

- If $G_{i} \notin \mathcal{F}$, we set $\mathcal{G}^{i}=\mathcal{G}^{i-1} \backslash\{G\}$ and $\mathcal{H}_{1}^{i}=\mathcal{H}_{1}^{i-1}, \mathcal{H}_{2}^{i}=\mathcal{H}_{2}^{i-1}$.

- If $G_{i} \in \mathcal{F}$, and the $\mathcal{G}^{i-1}$-weight of $G_{i}$ is more than $\varepsilon^{2} n^{t}$, then we again take the largest antichain $\mathcal{A}^{i}$ in $U_{\mathcal{G}^{i-1}}\left(G_{i}\right)$ with the smallest index in our fixed ordering of $2^{2^{[n]}}$ and set $\mathcal{G}^{i}=\mathcal{G}^{i-1} \backslash\left(\mathcal{A}^{i} \cup\left\{G_{i}\right\}\right), \mathcal{H}_{2}^{i}=\mathcal{H}_{2}^{i-1} \cup\left[\left(\mathcal{A}^{i} \cap \mathcal{F}\right) \cup\left\{G_{i}\right\}\right]$.

- If $G_{i} \in \mathcal{F}$, and the $\mathcal{G}^{i-1}$-weight of $G_{i}$ is at most $\varepsilon^{2} n^{t}$, then Phase II and the algorithm is ended, set $\mathcal{H}_{2}=\mathcal{H}_{2}^{i-1}$ and define $g\left(\mathcal{H}_{1} \cup \mathcal{H}_{2}\right)=\mathcal{G}^{i-1}$.

Observe the following:

- whenever we include sets in $\mathcal{H}_{1}^{i}$, then the number of such sets is at most $r+1$ (as $\mathcal{F}$ is

$\vee_{r+1}$-free), and the number of sets removed from $\mathcal{G}^{i-1}$ is at least $n^{t+0.9}$, so

$$
\left|\mathcal{H}_{1}\right| \leqslant(r+1) 2^{n} / n^{t+0.9}
$$

- at the end of Phase I, $\mathcal{G}^{i}$ does not contain any induced copies of $\vee_{n^{t+0.9}}$, so, by Theorem 9,

$$
\left|f\left(\mathcal{H}_{1}\right)\right|=\left|\mathcal{G}^{i}\right| \leqslant(t+1+\varepsilon)\left(\begin{array}{c}
n \\
\lfloor n / 2\rfloor
\end{array}\right)
$$

- the above two bullet points and the threshold for Phase II imply that

$$
\left|\mathcal{H}_{2}\right| \leqslant \frac{r+1}{\varepsilon^{2} n^{t}}\left|f\left(\mathcal{H}_{1}\right)\right| \leqslant(r+1) \frac{t+2}{\varepsilon^{2} n^{t}}\left(\begin{array}{c}
n \\
\lfloor n / 2\rfloor
\end{array}\right) ;
$$

- Theorem 9 implies that at the end of Phase II,

$$
\left|g\left(\mathcal{H}_{1} \cup \mathcal{H}_{2}\right)\right|=\left|\mathcal{G}^{i}\right| \leqslant(t+\varepsilon)\left(\begin{array}{c}
n \\
\lfloor n / 2\rfloor
\end{array}\right)
$$


All what remains to prove is that the functions $f$ and $g$ are well defined, i.e., if for two distinct $\vee_{r+1}$-free families $\mathcal{F}$ and $\mathcal{F}^{\prime}$ the algorithm outputs the same $\mathcal{H}_{1}$, then $f\left(\mathcal{H}_{1}\right)$ is defined the same, and if in addition $\mathcal{H}_{1} \cup \mathcal{H}_{2}$ is the same for both runs of the algorithm, then so is $g\left(\mathcal{H}_{1} \cup \mathcal{H}_{2}\right)$. We claim more: the families $\mathcal{G}^{i}, \mathcal{H}_{1}^{i}, \mathcal{H}_{2}^{i}$ are the same for both runs for all values of $i=0,1, \ldots$ This is certainly true for $i=0$. Then by induction, if this holds for some $i$, then $G^{i}$ is the same for both runs. Therefore, due to the fixed ordering of $2^{[n]}$, the algorithm considers the same set $G_{i+1} \in \mathcal{G}^{i}$ in step $i+1$. As $G_{i+1}$ will be removed from $\mathcal{G}^{i}$ in all cases, therefore it cannot happen that $G_{i+1}$ belongs to exactly one of $\mathcal{F}, \mathcal{F}^{\prime}$. If $G_{i+1} \notin \mathcal{F}, \mathcal{F}^{\prime}$, then $G_{i+1}$ is removed from $\mathcal{G}^{i}$ and nothing happens to $\mathcal{H}_{1}, \mathcal{H}_{2}$ so the claim is true for $i+1$. If $G_{i+1} \in \mathcal{F} \cap \mathcal{F}^{\prime}$, then, due to the fixed ordering of $2^{2^{[n]}}$, the antichain $\mathcal{A}^{i+1}$ is defined the same for both runs. This immediately yields that $\mathcal{G}^{i+1}$ is defined the same for both runs. Also, as for any $A \in \mathcal{A}^{i+1}$ either $A$ becomes a set of $\mathcal{H}_{1}, \mathcal{H}_{2}$ in this step or never, and in the end these sets are the same, therefore they must be the same after step $i+1$.

\section{Proofs}

\section{1 $\vee_{r+1}$-free families and consequences - Theorem 4, 6, and 7}

In this subsection we present the proofs of our theorems concerning $\vee_{r+1}$-free and $K_{s, 1, t^{-}}$ free families. We restate the theorems here for convenience.

Theorem 4. For any $s, t \in \mathbb{N}$ and $\varepsilon>0$ there exist $n_{0}=n_{\varepsilon, s, t}$ and $\delta>0$ such that any $\mathcal{F} \subseteq 2^{[n]}$ of size at least $(2+\varepsilon)\left(\begin{array}{c}n \\ \lfloor n / 2\rfloor\end{array}\right)$ with $n \geqslant n_{0}$ contains at least $\delta n^{s+t}\left(\begin{array}{c}n \\ \lfloor n / 2\rfloor\end{array}\right)$ induced copies of $K_{s, 1, t}$.

Proof. Let $\mathcal{F} \subseteq 2^{[n]}$ be a family of sets of size $(2+\varepsilon)\left(\begin{array}{c}n \\ \lfloor n / 2\rfloor\end{array}\right)$. Let $\mathcal{D}$ be the family of those elements of $\mathcal{F}$ that are not the maximal element of an induced $\wedge_{\varepsilon n / 10}$ and $\mathcal{U}$ the family of those that are not the minimal element of an induced $\vee_{\varepsilon n / 10}$. By Theorem 8 , we have $|\mathcal{D}|,|\mathcal{U}| \leqslant(1+4 \varepsilon / 10)\left(\begin{array}{c}n \\ \lfloor n / 2\rfloor\end{array}\right)$, thus $|\mathcal{F} \backslash(\mathcal{D} \cup \mathcal{U})| \geqslant \frac{\varepsilon}{5}\left(\begin{array}{c}n \\ \lfloor n / 2\rfloor\end{array}\right)$. Taking sets from $\mathcal{F} \backslash(\mathcal{D} \cup \mathcal{U})$ to play the role of the middle element of $K_{s, 1, t}$, by definition of $\mathcal{D}$ and $\mathcal{U}$, we obtain at least $\frac{\varepsilon}{5}\left(\begin{array}{c}n \\ \lfloor n / 2\rfloor\end{array}\right)\left(\begin{array}{c}\varepsilon n / 10 \\ s\end{array}\right)\left(\begin{array}{c}\varepsilon n / 10 \\ t\end{array}\right)$ copies of $K_{s, 1, t}$.

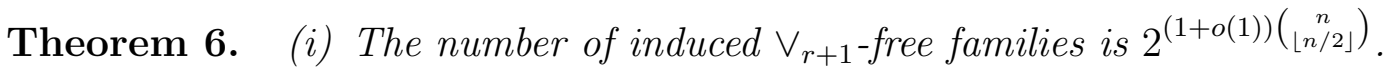

(ii) The number of induced $K_{s, 1, t}$-free families in $2^{[n]}$ is $2^{(2+o(1))\left(\begin{array}{c}n \\ \lfloor n / 2\rfloor\end{array}\right)}$.

Proof. To prove (i), we apply our container lemma, Theorem 10 with $t=1$. It shows that for every induced $\vee_{r+1}$-free family $\mathcal{F}$, there exist $\mathcal{H}_{1}, \mathcal{H}_{2}$ and $g\left(\mathcal{H}_{1} \cup \mathcal{H}_{2}\right)$ such that $\mathcal{H}_{1}$ and $\mathcal{H}_{2}$ are disjoint, $\left|\mathcal{H}_{1} \cup \mathcal{H}_{2}\right| \leqslant(r+1) \frac{3}{\varepsilon^{2} n}\left(\begin{array}{c}n \\ \lfloor n / 2\rfloor\end{array}\right),\left|g\left(\mathcal{H}_{1} \cup \mathcal{H}_{2}\right)\right| \leqslant(1+\varepsilon)\left(\begin{array}{c}n \\ \lfloor n / 2\rfloor\end{array}\right)$ and $\mathcal{F} \subseteq \mathcal{H}_{1} \cup \mathcal{H}_{2} \cup g\left(\mathcal{H}_{1} \cup \mathcal{H}_{2}\right)$. Therefore, $\left|\mathcal{H}_{1} \cup \mathcal{H}_{2} \cup g\left(\mathcal{H}_{1} \cup \mathcal{H}_{2}\right)\right| \leqslant(1+2 \varepsilon)\left(\begin{array}{c}n \\ \lfloor n / 2\rfloor\end{array}\right)$. The number of subfamilies of such containers is at most $2^{(1+2 \varepsilon)\left(\begin{array}{c}n \\ \lfloor n / 2\rfloor\end{array}\right)}$, and the number of such containers is at most

$$
\left(\begin{array}{c}
2^{n} \\
(r+1) \frac{3}{\varepsilon^{2} n}\left(\begin{array}{c}
n \\
\lfloor n / 2\rfloor
\end{array}\right)
\end{array}\right) 2^{(r+1) \frac{3}{\varepsilon^{2} n}\left(\begin{array}{c}
n \\
\lfloor n / 2\rfloor
\end{array}\right)} .
$$


Indeed, the first term is an obvious upper bound on the number of families $\mathcal{H}_{1} \cup \mathcal{H}_{2}$, and the second term is an obvious upper bound on the number of ways to partition $\mathcal{H}_{1} \cup \mathcal{H}_{2}$ to $\mathcal{H}_{1}$ and $\mathcal{H}_{2}$. Using $\left(\begin{array}{c}n \\ \lfloor n / 2\rfloor\end{array}\right)=\Theta\left(\frac{1}{\sqrt{n}} 2^{n}\right)$ and $\left(\begin{array}{l}a \\ b\end{array}\right) \leqslant\left(\frac{e a}{b}\right)^{b}$, we obtain that the number of induced $\vee_{r+1}$-free families in $2^{[n]}$ is at most

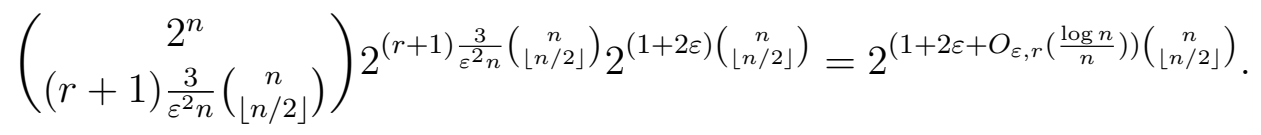

The lower bound follows from the fact that every subfamily of the middle level is $\vee_{r+1}$-free.

To prove (ii), observe that every induced $K_{s, 1, t}$ free family $\mathcal{F} \subseteq 2^{[n]}$ can be written as $\mathcal{F}=\mathcal{D} \cup \mathcal{U}$ such that $\mathcal{D}$ is induced $\wedge_{s}$-free and $\mathcal{U}$ is induced $\vee_{t}$-free. Indeed, let $\mathcal{D}$ be the family of those elements of $\mathcal{F}$ that are not the maximal element of an induced $\wedge_{s}$ and $\mathcal{U}$ be the family of those that are not the minimal element of an induced $\vee_{t}$. If $F \in \mathcal{F}$ does not belong to $\mathcal{D} \cup \mathcal{U}$, then $F$ with its $s$ subsets and its $t$ supersets form an induced $K_{s, 1, t}$, which contradicts the $K_{s, 1, t}$-free property of $\mathcal{F}$. By part (i), there are $2^{(1+o(1))\left(\begin{array}{c}n \\ \lfloor n / 2\rfloor\end{array}\right)}$ induced $\vee_{t}$-free families in $2^{[n]}$ and there are $2^{(1+o(1))\left(\begin{array}{c}n \\ \lfloor n / 2\rfloor\end{array}\right)}$ induced $\wedge_{s}$-free families in $2^{[n]}$. Therefore there are at most $2^{(2+o(1))\left(\begin{array}{c}n \\ \lfloor n / 2\rfloor\end{array}\right)}$ induced $K_{s, 1, t}$-free families in $2^{[n]}$. The lower bound immediately follows from the fact that every subfamily of the middle two levels is $K_{s, 1, t}$-free.

Theorem 7. If $p=\omega(1 / n)$, then the following are true.

(i) For any integer $r \geqslant 0$, the largest induced $\vee_{r+1}$-free family in $\mathcal{P}(n, p)$ has size $(1+$ $o(1)) p\left(\begin{array}{c}n \\ \lfloor n / 2\rfloor\end{array}\right)$ w.h.p..

(ii) For any pair $s, t \geqslant 1$ of integers, the largest induced $K_{s, 1, t}$-free family in $\mathcal{P}(n, p)$ has size $(2+o(1)) p\left(\begin{array}{c}n \\ \lfloor n / 2\rfloor\end{array}\right)$ w.h.p..

Proof. To prove (i), we again apply Theorem 10 with $t=1$. All calculations are very close to those in [12], which in turn are almost the same as those in [1], we include them for sake of completeness.

We will show that w.h.p. for every $\varepsilon>0$ and every $\vee_{r+1}$-free family $\mathcal{F}$ in $2^{[n]}$ of size at least $(1+\varepsilon) p\left(\begin{array}{c}n \\ \lfloor n / 2\rfloor\end{array}\right)$, not all sets of $\mathcal{F}$ remain in $\mathcal{P}(n, p)$. It is enough to prove the statement for $\varepsilon<\frac{1}{4}$ and set $\varepsilon_{1}=\varepsilon / 4$. For every such $\mathcal{F}$, Theorem 10 with $\varepsilon_{1}$ in the role of $\varepsilon$, gives us $\mathcal{H}_{1}=\mathcal{H}_{1}(\mathcal{F}), \mathcal{H}_{2}=\mathcal{H}_{2}(\mathcal{F})$ such that

- $\mathcal{H}_{1} \in\left(\begin{array}{c}2^{[n]} \\ \leqslant(r+1) 2^{n} n^{-1.9}\end{array}\right)$; therefore the number of possible $\mathcal{H}_{1}$ 's is at most

$$
\sum_{a \leqslant(r+1) 2^{n} n^{-1.9}}\left(\begin{array}{c}
2^{n} \\
a
\end{array}\right) .
$$

Clearly, we have $\mathbb{P}\left(\mathcal{H}_{1} \subseteq \mathcal{P}(n, p)\right)=p^{\left|\mathcal{H}_{1}\right|}$. 


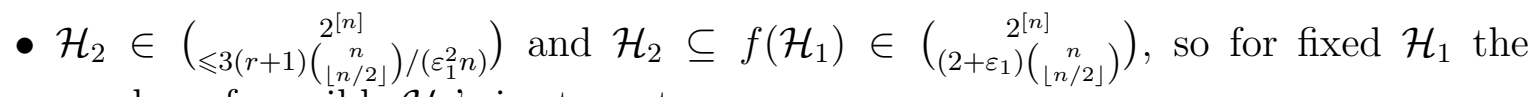
number of possible $\mathcal{H}_{2}$ 's is at most

$$
\left|\left(\begin{array}{c}
f\left(\mathcal{H}_{1}\right) \\
\leqslant 3(r+1)\left(\begin{array}{c}
n \\
\lfloor n / 2\rfloor
\end{array}\right) /\left(\varepsilon_{1}^{2} n\right)
\end{array}\right)\right| \leqslant \sum_{b \leqslant 3(r+1)\left(\begin{array}{c}
n \\
\lfloor n / 2\rfloor
\end{array}\right) /\left(\varepsilon_{1}^{2} n\right)}\left(\begin{array}{c}
3\left(\begin{array}{c}
n \\
\lfloor n / 2\rfloor
\end{array}\right) \\
b
\end{array}\right) .
$$

Also, $\mathbb{P}\left(\mathcal{H}_{2} \subseteq \mathcal{P}(n, p)\right)=p^{\left|\mathcal{H}_{2}\right|}$.

- For fixed $\mathcal{H}_{1}$ and $\mathcal{H}_{2}$ the corresponding $\mathcal{F}$ 's are all subfamilies of $\mathcal{H}_{1} \cup \mathcal{H}_{2} \cup g\left(\mathcal{H}_{1} \cup \mathcal{H}_{2}\right)$ and contain $\mathcal{H}_{1} \cup \mathcal{H}_{2}$.

1. Let $\mathcal{E}_{\mathcal{H}_{1}, \mathcal{H}_{2}}$ be the event that there exists any $\mathcal{F}$ with $\mathcal{H}_{1}(\mathcal{F})=\mathcal{H}_{1}, \mathcal{H}_{2}(\mathcal{F})=\mathcal{H}_{2}$, and $|\mathcal{F}| \geqslant(1+\varepsilon) p\left(\begin{array}{c}n \\ \lfloor n / 2\rfloor\end{array}\right)$, and

2. let $\mathcal{E}_{g\left(\mathcal{H}_{1} \cup \mathcal{H}_{2}\right)}$ be the event that $\left|\mathcal{P}(n, p) \cap g\left(\mathcal{H}_{1} \cup \mathcal{H}_{2}\right)\right| \geqslant(1+\varepsilon) p\left(\begin{array}{c}n \\ \lfloor n / 2\rfloor\end{array}\right)-\left|\mathcal{H}_{1} \cup \mathcal{H}_{2}\right|$ holds.

We bound the probability of the event $\mathcal{E}_{\mathcal{H}_{1}, \mathcal{H}_{2}}$ by the probability of the event $\mathcal{E}_{g\left(\mathcal{H}_{1} \cup \mathcal{H}_{2}\right)}$. Note that

$$
(1+\varepsilon) p\left(\begin{array}{c}
n \\
\lfloor n / 2\rfloor
\end{array}\right)-\left|\mathcal{H}_{1} \cup \mathcal{H}_{2}\right| \geqslant(1+\varepsilon / 2) p\left(\begin{array}{c}
n \\
\lfloor n / 2\rfloor
\end{array}\right)
$$

and

$$
\left|g\left(\mathcal{H}_{1} \cup \mathcal{H}_{2}\right)\right| \leqslant\left(1+\varepsilon_{1}\right)\left(\begin{array}{c}
n \\
\lfloor n / 2\rfloor
\end{array}\right) \leqslant(1+\varepsilon / 4)\left(\begin{array}{c}
n \\
\lfloor n / 2\rfloor
\end{array}\right) .
$$

Therefore, $\left|\mathcal{P}(n, p) \cap g\left(\mathcal{H}_{1} \cup \mathcal{H}_{2}\right)\right|$ is binomially distributed with

$$
\mathbb{E}\left(\left|\mathcal{P}(n, p) \cap g\left(\mathcal{H}_{1} \cup \mathcal{H}_{2}\right)\right|\right) \leqslant(1+\varepsilon / 4) p\left(\begin{array}{c}
n \\
\lfloor n / 2\rfloor
\end{array}\right),
$$

so by Chernoff's inequality we have

$$
\mathbb{P}\left(\mathcal{E}_{g\left(\mathcal{H}_{1}, \mathcal{H}_{2}\right)}\right) \leqslant \mathbb{P}\left[\left|\mathcal{P}(n, p) \cap g\left(\mathcal{H}_{1} \cup \mathcal{H}_{2}\right)\right| \geqslant(1+\varepsilon / 2) p\left(\begin{array}{c}
n \\
\lfloor n / 2\rfloor
\end{array}\right)\right] \leqslant e^{-\varepsilon^{2} p\left(\begin{array}{c}
n \\
\lfloor n / 2\rfloor
\end{array}\right) / 100} .
$$

Note that $\mathcal{H}_{1}, \mathcal{H}_{2}$, and $g\left(\mathcal{H}_{1} \cup \mathcal{H}_{2}\right)$ are disjoint, so the three events that $\mathcal{H}_{1} \subseteq$ $\mathcal{P}(n, p), \mathcal{H}_{2} \subseteq \mathcal{P}(n, p)$ and $\mathcal{E}_{g\left(\mathcal{H}_{1} \cup \mathcal{H}_{2}\right)}$ are independent. Hence the probability that for fixed $\mathcal{H}_{1}$ and $\mathcal{H}_{2}$ there is a corresponding large induced $\vee_{r+1}$-free family $\mathcal{F}$, is at most $p^{\left|\mathcal{H}_{1}\right|+\left|\mathcal{H}_{2}\right|} e^{-\varepsilon^{2} p\left(\begin{array}{c}n \\ \lfloor n / 2\rfloor\end{array}\right) / 100}$. Summing up for all possible $\mathcal{H}_{1}$ and $\mathcal{H}_{2}$, we obtain that the probability that there is an induced $\vee_{r+1}$-free family in $\mathcal{P}(n, p)$ of size $(1+\varepsilon) p\left(\begin{array}{c}n \\ \lfloor n / 2\rfloor\end{array}\right)$, is at most

$$
\sum_{0 \leqslant a \leqslant(r+1) n^{-1.9} 2^{n}} \sum_{0 \leqslant b \leqslant 3(r+1)\left(\begin{array}{c}
n \\
\lfloor n / 2\rfloor
\end{array}\right) /\left(\varepsilon_{1}^{2} n\right)}\left(\begin{array}{c}
2^{n} \\
a
\end{array}\right) p^{a}\left(\begin{array}{c}
3\left(\begin{array}{c}
n \\
\lfloor/ 2\rfloor
\end{array}\right) \\
b
\end{array}\right) p^{b} e^{-\varepsilon^{2} p\left(\begin{array}{c}
n \\
\lfloor n / 2\rfloor
\end{array}\right) / 100} .
$$


It is not hard to verify that the largest summand in the above sum belongs to the largest possible values of $a$ and $b$. Therefore the above expression is bounded from above by

$$
\begin{gathered}
(r+1) n^{-1.9} 2^{n} \frac{3(r+1)\left(\begin{array}{c}
n \\
\lfloor n / 2\rfloor
\end{array}\right)}{\varepsilon_{1}^{2} n}\left(\begin{array}{c}
2^{n} \\
(r+1) n^{-1.9} 2^{n}
\end{array}\right)\left(\begin{array}{c}
3\left(\begin{array}{c}
n \\
\lfloor n / 2\rfloor
\end{array}\right) \\
\frac{3(r+1)\left(\begin{array}{c}
n \\
\lfloor n / 2\rfloor
\end{array}\right)}{\left(\varepsilon_{1}^{2} n\right)}
\end{array}\right) \times \\
\times e^{-\varepsilon^{2} p\left(\begin{array}{c}
n \\
\lfloor n / 2\rfloor
\end{array}\right) / 100} p^{(r+1) n^{-1.9} 2^{n}} p^{3(r+1)\left(\begin{array}{c}
n \\
\lfloor n / 2\rfloor
\end{array}\right) /\left(\varepsilon^{2} n\right)} .
\end{gathered}
$$

Observe that

$$
(r+1) n^{-1.9} 2^{n} 3(r+1)\left(\begin{array}{c}
n \\
\lfloor n / 2\rfloor
\end{array}\right) /\left(\varepsilon_{1}^{2} n\right) \leqslant e^{O(n)} \leqslant e^{\varepsilon^{2} p\left(\begin{array}{c}
n \\
\lfloor n / 2\rfloor
\end{array}\right) / 400} .
$$

Also, using $\left(\begin{array}{l}n \\ k\end{array}\right) \leqslant\left(\frac{e n}{k}\right)^{k}$ and $p\left(\begin{array}{c}n \\ \lfloor n / 2\rfloor\end{array}\right)=\omega\left(n^{-1.5} 2^{n}\right)$, we have

$$
\left(\begin{array}{c}
2^{n} \\
(r+1) n^{-1.9} 2^{n}
\end{array}\right) p^{(r+1) n^{-1.9} 2^{n}} \leqslant\left(e n^{1.9} p\right)^{(r+1) n^{-1.9} 2^{n}} \leqslant e^{O\left(n^{-1.9} 2^{n} \ln n\right)} \leqslant e^{\varepsilon^{2} p\left(\begin{array}{c}
n \\
\lfloor n / 2\rfloor
\end{array}\right) / 400} .
$$

Finally, by the same reasoning we have

$$
\begin{aligned}
\left(\begin{array}{c}
3\left(\begin{array}{c}
n \\
\lfloor n / 2\rfloor
\end{array}\right) \\
3(r+1)\left(\begin{array}{c}
n \\
\lfloor n / 2\rfloor
\end{array}\right) /\left(\varepsilon_{1}^{2} n\right)
\end{array}\right) p^{3(r+1)\left(\begin{array}{c}
n \\
\lfloor n / 2\rfloor
\end{array}\right) /\left(\varepsilon_{1}^{2} n\right)} & \leqslant\left(e \varepsilon_{1}^{2} n p\right)^{3(r+1)\left(\begin{array}{c}
n \\
\lfloor n / 2\rfloor
\end{array}\right) /\left(\varepsilon_{1}^{2} n\right)} \\
& \leqslant e^{3(r+1)\left(\begin{array}{c}
n \\
\lfloor n / 2\rfloor
\end{array}\right) p \frac{\ln (n p)}{\left(\varepsilon^{2} n p\right)}} \leqslant e^{\varepsilon^{2} p\left(\begin{array}{c}
n \\
\lfloor n / 2\rfloor
\end{array}\right) / 400} .
\end{aligned}
$$

Therefore, the probability there is an induced $\vee_{r+1}$-free family in $\mathcal{P}(n, p)$ of size $(1+$ $\varepsilon) p\left(\begin{array}{c}n \\ \lfloor n / 2\rfloor\end{array}\right)$ is at most $e^{-\varepsilon^{2} p\left(\begin{array}{c}n \\ \lfloor n / 2\rfloor\end{array}\right) / 400}=o(1)$, as required.

To prove (ii), let $\mathcal{F} \subseteq \mathcal{P}(n, p)$ be an induced $K_{s, 1, t}$-free family. As in the proof of Theorem 6 (ii), let us consider the partition $\mathcal{F}=\mathcal{D} \cup \mathcal{U}$ with $\mathcal{D}=\left\{F \in \mathcal{F}: \nexists F_{1}, F_{2}, \ldots, F_{s}\right.$ an antichain with $\left.F_{i} \subsetneq F\right\}$ and $\mathcal{U}=\left\{F \in \mathcal{F}: \nexists F_{1}, F_{2}, \ldots, F_{t}\right.$ an antichain with $\left.F_{i} \supsetneq F\right\}$. By part (i) of this theorem, both $\mathcal{D}$ and $\mathcal{U}$ are of size at most $(1+o(1)) p\left(\begin{array}{c}n \\ \lfloor n / 2\rfloor\end{array}\right)$ and thus $|\mathcal{F}| \leqslant(2+o(1)) p\left(\begin{array}{c}n \\ \lfloor n / 2\rfloor\end{array}\right)$.

\subsection{Results on trees - Theorem 2 and 3}

Lemma 11. Let $\vec{T}$ be a directed tree with $t$ edges that does not contain directed paths of length 2. Then there exists a $\delta>0$ such that the following holds: if $f(m)$ tends to infinity with $m$ and $\vec{G}_{m}$ is a directed graph on $m$ vertices with $f(m) \cdot m$ edges, then $\vec{G}$ contains $\delta f(m)^{t} m$ copies of $\vec{T}$.

Proof. It is a well-known fact that every undirected graph contains a cut (i.e., a partition of its vertices into two and the edges between the parts) that contains at least half of its edges. This fact easily implies that the vertex set of $\vec{G}_{m}$ can be partitioned into $A \cup B$ such that there exist $\frac{f(m) m}{4}$ edges pointing from $A$ to $B$. Let $\vec{H}$ denote this directed 
subgraph of $\vec{G}_{m}$. We call the number of edges incident to a vertex degree, no matter what the direction of the edge is. We claim that $\vec{H}$ has a subgraph with minimum degree at least $\frac{f(m)}{5}$ and with at least $\frac{f(m) m}{20}$ edges. Indeed, let us remove vertices of degree less than $\frac{f(m)}{5}$, then we remove the vertices that have degree less than $\frac{f(m)}{5}$ in the resulting graph, and so on. We stop when we obtain a graph with minimum degree at least $\frac{f(m)}{5}$. Altogether we removed at most $m$ vertices, thus less than $\frac{f(m) m}{5}$ edges.

Based on these properties, we can embed $\vec{T}$ to $\vec{G}_{m}$ greedily as follows: we fix an ordering $e_{1}, e_{2}, \ldots, e_{t}$ of the edges of $\vec{T}$ such that $e_{1}, \ldots, e_{j}$ is a tree for every $j=1,2, \ldots, t$ and then embed $e_{i}$ to an edge of $\vec{H}_{t-i}$. There are at least $\frac{f(m) m}{20}$ choices for the image of $e_{1}$ and at least $\frac{f(m)}{5}-i$ choices for the image of $e_{i}$ if $i \geqslant 2$.

Theorem 2. Let $T$ be any height 2 tree poset of $t+1$ elements. Then for any $\varepsilon>0$ there exist $\delta>0$ and $n_{0}$ such that for any $n \geqslant n_{0}$ any family $\mathcal{F} \subseteq 2^{[n]}$ of size $|\mathcal{F}| \geqslant(1+\varepsilon)\left(\begin{array}{c}n \\ \lfloor n / 2\rfloor\end{array}\right)$ contains at least $\delta n^{t}\left(\begin{array}{c}n \\ \lfloor n / 2\rfloor\end{array}\right)$ copies of $T$.

Proof. For a family $\mathcal{F} \subseteq 2^{[n]}$ of size at least $(1+\varepsilon)\left(\begin{array}{c}n \\ \lfloor n / 2\rfloor\end{array}\right)$, let us consider its directed comparability graph $\vec{G}_{\mathcal{F}}$. This is the graph with vertex set $\mathcal{F}$ where $\overrightarrow{F G}$ is an edge if and only if $F \subsetneq G$. By Theorem $1, \vec{G}_{\mathcal{F}}$ contains at least $\varepsilon \frac{n}{4}\left(\begin{array}{c}n \\ \lfloor n / 2\rfloor\end{array}\right)$ edges and thus Lemma 11 can be applied with $m=\left(\begin{array}{c}n \\ \lfloor n / 2\rfloor\end{array}\right)$ and $f(m)=\varepsilon \frac{n}{4}$.

Proposition 12. For any monotone tree poset $T$, we have $x(T)=|T|-1+\sum(h(T)-r(\ell))$, where $h(T)$ denotes the height of $T$, the summation is over all leaves $\ell$ of $T$, and $r(\ell)$ is the rank of $\ell$, i.e., its distance from the root in the Hasse diagram of $T$ plus 1.

Proof. Let us generate an embedding $i$ of $T$ into the $h(T)$ middle levels. Assume without loss of generality that $T$ is upward monotone, thus the root must be mapped to the lowest of the middle levels. We will refer to this level as the first middle level, and analogously the level above it is the second middle level, and the $k$ th middle level is above the first level by $k-1$.

Observe that there are $(1+o(1))\left(\begin{array}{c}n \\ \lfloor n / 2\rfloor\end{array}\right)$ ways to pick the image of the root. Then we define $i$ by going through the elements of $T$ in a non-decreasing order with respect to the rank $r(p)$. That means that for every element $p$, when we decide where to embed it, we have already embedded its predecessor, the (unique) element $p^{\prime}$ that is smaller than $p$ such that there is no element $p^{\prime \prime}$ with $p^{\prime}<p^{\prime \prime}<p$.

If the predecessor $p^{\prime}$ of $p$ is mapped to the $j$ th middle level and we want to map $p$ to the $k$ th middle level, then the number of possibilities is $\Theta\left(n^{k-j}\right)$. Let $f_{i}: T \rightarrow[h(T)]$ be a function defined by $f_{i}(p)=k$ if $i(p)$ is on the $k$ th middle level. For a given $f: T \rightarrow[h(T)]$, the number of embeddings $i$ with $f_{i}=f$ is $\Theta\left(n^{\sum_{p \in T}\left(f(p)-f\left(p^{\prime}\right)\right)}\right)$, where $p^{\prime}$ is the predecessor of $p$ and the summation goes over all elements of $P$ apart from the root. Therefore, to obtain $x(T)$, we need to maximize $\Theta\left(n^{\sum_{p \in P}\left(f(p)-f\left(p^{\prime}\right)\right)}\right)$. Clearly, when embedding a leaf $\ell$, we must have $f(\ell)=h(T)$. Finally, observe that if for some non-leaf $p$, we have $f(p)>f\left(p^{\prime}\right)+1$, then changing $f(p)$ to $f\left(p^{\prime}\right)+1$ cannot decrease the sum (and strictly 
increases it, if $p$ has at least two children). This shows that a function $f$ that maximizes the sum must satisfy $f(\ell)=h(T)$ for all leaves, and $f(p)=r(p)$ for all non-leaves.

Lemma 13. Assume that the families $2^{[n]} \supset \mathcal{F}_{1} \supset \mathcal{F}_{2} \supset \cdots \supset \mathcal{F}_{h}$ satisfy the following properties for some $\delta_{1}, \delta_{2}, \delta_{3}>0$.

(i) $\left|\mathcal{F}_{h}\right| \geqslant \delta_{1}\left(\begin{array}{c}n \\ \lfloor n / 2\rfloor\end{array}\right)$.

(ii) For every $i=2,3, \ldots, h$ and $F \in \mathcal{F}_{i}$ there exist at least $\delta_{2} n$ sets $F^{\prime} \in \mathcal{F}_{i-1}$ with $F \subsetneq F^{\prime}$.

(iii) For every $i=2,3, \ldots, h$ and $F \in \mathcal{F}_{i}$ there exist at least $\delta_{3} n^{i-1}$ sets $F^{\prime} \in \mathcal{F}_{1}$ with $F \subsetneq F^{\prime}$.

Then for any upward monotone tree poset $T$ of height $h(T)=h, \mathcal{F}_{1}$ contains at least $\delta n^{x(T)}\left(\begin{array}{c}n \\ \lfloor n / 2\rfloor\end{array}\right)$ copies of $T$, where $\delta>0$ is a constant depending on $\delta_{1}, \delta_{2}, \delta_{3}$ and $|T|$.

Proof. We generate embeddings of $T$ as follows: we embed elements of $T$ according to their rank. The root of $T$ can be embedded to any set $F \in \mathcal{F}_{h}$. By i), we have at least $\delta_{1}\left(\begin{array}{c}n \\ \lfloor n / 2\rfloor\end{array}\right)$ choices.

Any non-leaf element $x \in T$ with its predecessor embedded to $F_{i} \in \mathcal{F}_{i}$ can be embedded to any set $F^{\prime} \in \mathcal{F}_{i-1}$ with $F_{i} \subsetneq F^{\prime}$ that has not yet been used by the embedding. By ii), we have at least $\delta_{2} n-|T|$ choices. If $x$ is of rank $r(x)$ then this process will embed it to $\mathcal{F}_{h+1-r(x)}$.

If $\ell$ is a leaf vertex of rank $r(\ell)$ in $T$, then its predecessor is embedded into some $F \in \mathcal{F}_{h+2-r(\ell)}$. We can embed $\ell$ to any superset of $F$ that has not yet been used. By iii), there exist at least $\delta_{3} n^{h+1-r(\ell)}-|T|$ such sets.

Proposition 12 yields that the exponent of $n$ in the number of embeddings generated this way will be exactly $x(T)$. (We get a factor of $n$ for all vertices except for the root, and an additional $n^{h-r(\ell)}$ for leaves $\ell$.) A copy corresponds to at most $|T|$ ! embeddings.

Theorem 3. For any monotone tree poset $T$ and $\varepsilon>0$, there exist $\delta>0$ and $n_{0}$ such that for any $n \geqslant n_{0}$ any family $\mathcal{F} \subseteq 2^{[n]}$ of size $|\mathcal{F}| \geqslant(h(T)-1+\varepsilon)\left(\begin{array}{c}n \\ \lfloor n / 2\rfloor\end{array}\right)$ contains at least $\delta n^{x(T)}\left(\begin{array}{c}n \\ \lfloor n / 2\rfloor\end{array}\right)$ copies of $T$.

Proof. Throughout the proof we can assume that $n$ is sufficiently large. Let $h:=h(T)$. To prove the theorem, we will find families $\mathcal{F} \supseteq \mathcal{F}_{1} \supset \mathcal{F}_{2} \supset \cdots \supset \mathcal{F}_{h}$ that satisfy the conditions of Lemma 13 for sufficiently small $\left(\delta_{1}, \delta_{2}, \delta_{3}\right)$ depending on $\varepsilon$ and $T$. Let $\mathcal{F}_{1}=\left\{G \in \mathcal{F}:|| G|-n / 2|<n^{2 / 3}\right\}$. Then $\left|\mathcal{F} \backslash \mathcal{F}_{1}\right| \leqslant\left|\left\{G \subseteq[n]:|| G|-n / 2| \geqslant n^{2 / 3}\right\}\right| \leqslant$ $O\left(\left(\begin{array}{c}n \\ \lfloor n / 2\rfloor\end{array}\right)\right)$.

For $i=2,3, \ldots, h$ we define $\mathcal{F}_{i}$ as follows. Let $\varepsilon^{\prime}:=\varepsilon / 2 h$. A set $F \in \mathcal{F}_{i-1}$ is in $\mathcal{F}_{i}$ if the expected number of sets of $\mathcal{F}_{i-1}$ in a random chain between $F$ and $[n]$ is at least $1+\varepsilon^{\prime}$. ( $F$ counts for all chains.)

Let $\mathcal{G}_{i}:=\mathcal{F}_{i-1} \backslash \mathcal{F}_{i}$. We partition the $n$ ! full chains from $\emptyset$ to $[n]$ as follows. Let $\mathbf{C}_{G}$ denote the collection of those chains whose smallest member from $\mathcal{G}_{i}$ is $G$. Let $\mathbf{C}_{0}$ denote 
the collection of those full chains that avoid $\mathcal{G}_{i}$. If we take a random full chain from any of these collections, the expected value of members of $\mathcal{G}_{i}$ in it is at most $1+\varepsilon^{\prime}$. Therefore the expected value taken over all $n$ ! full chains is also at most $1+\varepsilon^{\prime}$. This means that $\lambda_{n}\left(\mathcal{G}_{i}\right):=\sum_{F \in \mathcal{G}_{i}} \frac{1}{\left(\begin{array}{c}n \\ |F|\end{array}\right)} \leqslant 1+\varepsilon^{\prime}$ and therefore $\left|\mathcal{G}_{i}\right| \leqslant\left(1+\varepsilon^{\prime}\right)\left(\begin{array}{c}n \\ \lfloor n / 2\rfloor\end{array}\right)$. These inequalities for $i=2,3, \ldots, h$ together imply i) of Lemma 13 , if $\delta_{1}<\varepsilon / 2$.

Consider a set $F \in \mathcal{F}_{i-1}$. If $F$ has at most $\delta_{2} n$ supersets of size between $|F|+1$ and $|F|+h-1$ in $\mathcal{F}_{i-1}$ and at most $\left(\delta_{2}+\delta_{3}\right) n^{h}$ supersets in all of $\mathcal{F}_{i-1}$, then the expected value of elements of $\mathcal{F}_{i-1}$ in a chain from $F$ to $[n]$ is

$$
\sum_{F \subsetneq F^{\prime} \in \mathcal{F}_{i-1}} \frac{1}{\left(\begin{array}{c}
n-|F| \\
\left|F^{\prime} \backslash F\right|
\end{array}\right)} \leqslant \frac{\delta_{2} n}{n-|F|}+\frac{\left(\delta_{2}+\delta_{3}\right) n^{h}}{\left(\begin{array}{c}
n-|F| \\
h
\end{array}\right)} \leqslant 3 \delta_{2}+3^{h} h !\left(\delta_{2}+\delta_{3}\right)
$$

which is smaller than $\varepsilon^{\prime}$ if $\delta_{2}$ and $\delta_{3}$ are a sufficiently small positive numbers compared to $h$ and $\varepsilon^{\prime}$. This means that any $G \in \mathcal{F}_{i}$ has either at least $\delta_{2} n$ proper supersets of size at most $|G|+h-1$ in $\mathcal{F}_{i-1}$ or at least $\left(\delta_{2}+\delta_{3}\right) n^{h}$ supersets in all of $\mathcal{F}_{i-1}$.

The above statement trivially implies ii) of Lemma 13. We show that iii) of Lemma 13 follows as well. Let $F \in \mathcal{F}_{i}$ for some $2 \leqslant i \leqslant h$. We need to find at least $\delta_{3} n^{i-1}$ supersets of $F$ in $\mathcal{F}_{1}$. If it has $\left(\delta_{2}+\delta_{3}\right) n^{h}$ supersets in $\mathcal{F}_{i-1}$ then we are obviously done, so assume that this is not the case. We define a directed graph as follows. $F$ has at least $\delta_{2} n$ supersets of size at most $|G|+h-1$ in $\mathcal{F}_{i-1}$, let us direct an edge from $F$ to all of them. Each of these sets will have at least $\delta_{2} n$ supersets of size at most $|G|+2(h-1)$ in $\mathcal{F}_{i-2}$, direct an edge from the subset to the supersets. Continue this operation until we reach $\mathcal{F}_{1}$. Now we have at least $\left(\delta_{2} n\right)^{i-1}$ directed paths from $G$ to members of $\mathcal{F}_{1}$. All these members are of size at most $|G|+(i-1)(h-1)$, therefore the number of paths leading to a single one of them is at most $((i-1)(h-1))^{i-1}<h^{2 h}$. Thus we found at least $\frac{\left(\delta_{2} n\right)^{i-1}}{h^{2 h}}$ supersets of $G$ in $\mathcal{F}_{1}$. This is more than $\delta_{3} n^{i-1}$ if $\delta_{3}$ is sufficiently small compared to $\delta_{2}$ and $h$.

\subsection{Diamond-free families - Theorem 5}

Let us recall that $m_{s}=\left\lceil\log _{2}(s+2)\right\rceil$ and $m_{s}^{*}=\min \left\{m: s \leqslant\left(\begin{array}{c}m \\ \lceil m / 2\rceil\end{array}\right)\right\}$ and that for any integer $s \geqslant 2$, we have $x\left(D_{s}\right)=m_{s}$ and $x^{*}\left(D_{s}\right)=m_{s}^{*}$.

Theorem 5. (i) If $s \in\left[2^{m_{s}-1}-1,2^{m_{s}}-\left(\begin{array}{c}m_{s} \\ \left\lceil\frac{m_{s}}{2}\right\rceil\end{array}\right)-1\right]$, then for any $\varepsilon>0$ there exists $a \delta>0$ such that every $\mathcal{F} \subseteq 2^{[n]}$ with $|\mathcal{F}| \geqslant\left(m_{s}+\varepsilon\right)\left(\begin{array}{c}n \\ \lfloor n / 2\rfloor\end{array}\right)$ contains at least $\delta \cdot n^{m_{s}-0.5}\left(\begin{array}{c}n \\ \lfloor n / 2\rfloor\end{array}\right)$ copies of $D_{s}$.

(ii) For any $\varepsilon>0$ there exists a $\delta>0$ such that every $\mathcal{F} \subseteq 2^{[n]}$ with $|\mathcal{F}| \geqslant(4+\varepsilon)\left(\begin{array}{c}n \\ \lfloor n / 2\rfloor\end{array}\right)$ contains at least $\delta \cdot n^{3.5}\left(\begin{array}{c}n \\ \lfloor n / 2\rfloor\end{array}\right)$ induced copies of $D_{4}$.

(iii) For any constant $c$ with $1 / 2<c<1$ there exists an integer $s_{c}$ such that if $s \geqslant s_{c}$ and $s \leqslant c\left(\begin{array}{c}m_{s}^{*} \\ \left.m_{s}^{*} / 2\right\rfloor\end{array}\right)$, then the following holds: for any $\varepsilon>0$ there exists a $\delta>0$ such that every $\mathcal{F} \subseteq 2^{[n]}$ with $|\mathcal{F}| \geqslant\left(m_{s}^{*}+\varepsilon\right)\left(\begin{array}{c}n \\ \lfloor n / 2\rfloor\end{array}\right)$ contains at least $\delta \cdot n^{m_{s}^{*}-0.5}\left(\begin{array}{c}n \\ \lfloor n / 2\rfloor\end{array}\right)$ induced copies of $D_{s}$. 
Proof. To prove (i), let us fix $\varepsilon>0$ and let $\mathcal{F} \subseteq 2^{[n]}$ such that $|\mathcal{F}| \geqslant\left(m_{s}+\varepsilon\right)\left(\begin{array}{c}n \\ \lfloor n / 2\rfloor\end{array}\right)$. Let $\ell$ be the maximum integer such that $\left(\begin{array}{c}n \\ \ell\end{array}\right) \leqslant \frac{\varepsilon}{8 \sqrt{n}}\left(\begin{array}{c}n \\ \lfloor n / 2\rfloor\end{array}\right)$. Then by Lemma 8, we have $\left|\left(\begin{array}{c}{[n]} \\ \leqslant \ell\end{array}\right) \cup\left(\begin{array}{c}{[n]} \\ \geqslant n-\ell\end{array}\right)\right| \leqslant \frac{\varepsilon}{2}\left(\begin{array}{c}n \\ \lfloor n / 2\rfloor\end{array}\right)$ and it is easy to verify that $\left(\begin{array}{c}n \\ \ell\end{array}\right) \geqslant \frac{\varepsilon}{10 \sqrt{n}}\left(\begin{array}{c}n \\ \lfloor n / 2\rfloor\end{array}\right)$ if $n$ is large enough. This implies that

1. $\mathcal{F}^{\prime}=\mathcal{F} \backslash\left(\left(\begin{array}{c}{[n]} \\ \leqslant \ell\end{array}\right) \cup\left(\begin{array}{c}{[n]} \\ \geqslant n-\ell\end{array}\right)\right)$ is of size at least $\left(m_{s}+\varepsilon / 2\right)\left(\begin{array}{c}n \\ \lfloor n / 2\rfloor\end{array}\right)$, in particular $\sum_{F \in \mathcal{F}}|F| !(n-|F|) ! \geqslant\left(m_{s}+\varepsilon / 2\right) n !$

2. for any $F \in \mathcal{F}^{\prime}$, we have $|F| !(n-\mid F) ! \leqslant \frac{10}{\varepsilon} \sqrt{n}\lfloor n / 2\rfloor !\lceil n / 2\rceil$ !.

We are going to count pairs $(F, \mathcal{C})$ with $F \in \mathcal{F}^{\prime} \cap \mathcal{C}$ and $\mathcal{C}$ is a maximal chain in $[n]$. On the one hand, using the first point above, this is clearly $\sum_{F \in \mathcal{F}}|F| !(n-|F|) ! \geqslant\left(m_{s}+\varepsilon / 2\right) n$ !. To count the pairs in another way, we will use the min-max partition of the maximal chains. Let $\mathbf{C}_{n}$ denote the set of all $n$ ! maximal chains in $[n]$, and let us partition $\mathbf{C}_{n}$ into $\cup_{F, F^{\prime}} \mathbf{C}_{F, F^{\prime}}$, where $F, F^{\prime}$ run through all pairs $F \subseteq F^{\prime}$ in $\mathcal{F}^{\prime}$ and

$$
\mathbf{C}_{F, F^{\prime}}:=\left\{\mathcal{C} \in \mathbf{C}_{n}: F \text { is minimal in } \mathcal{F}^{\prime} \cap \mathcal{C}, F^{\prime} \text { is maximal in } \mathcal{F}^{\prime} \cap \mathcal{C}\right\} .
$$

Those maximal chains that do not contain any $F \in \mathcal{F}^{\prime}$ are gathered in $\mathbf{C}_{\emptyset}$. For any pair $F \subseteq F^{\prime}$ in $\mathcal{F}^{\prime}$ let us write $b\left(F, F^{\prime}\right)=\left|\mathcal{F}^{\prime} \cap\left\{G: F \subsetneq G \subsetneq F^{\prime}\right\}\right|$. Observe that the number of copies of $D_{s}$ in $\mathcal{F}^{\prime}$ is at least $\sum_{F, F^{\prime}}\left(\begin{array}{c}b\left(F, F^{\prime}\right) \\ s\end{array}\right)$, in particular this number is at least $\left|\left\{\left(F, F^{\prime}\right): b\left(F, F^{\prime}\right) \geqslant s\right\}\right|$. Finally, let $\mathbf{C}_{j}=\bigcup_{b\left(F, F^{\prime}\right)=j} \mathbf{C}_{F, F^{\prime}}$ and $\mathbf{C}_{<s}=\mathbf{C}_{\emptyset} \cup \bigcup_{j=0}^{s-1} \mathbf{C}_{j}$ and $\mathbf{C}_{\geqslant s}=\mathbf{C}_{n} \backslash \mathbf{C}_{<s}$.

As $b\left(F, F^{\prime}\right)<s$ is equivalent to $\left[F, F^{\prime}\right] \cap \mathcal{F}^{\prime}$ being $D_{s}$-free, using Lemma 9 , we obtain that for any $F \subseteq F^{\prime}$ with $b\left(F, F^{\prime}\right)<s$, the number of pairs $(G, \mathcal{C})$ with $G \in \mathcal{F}^{\prime} \cap \mathcal{C}$ and $\mathcal{C}$ a maximal chain in $\left[F, F^{\prime}\right]$, is at most $m_{s}\left|F^{\prime} \backslash F\right|$ !. Let $\alpha_{F}$ denote the number of maximal chains from $\emptyset$ to $F$ that do not contain any other element of $\mathcal{F}^{\prime}$ than $F$ and let $\beta_{F^{\prime}}$ denote the number of maximal chains from $F^{\prime}$ to $[n]$ that do not contain any other element of $\mathcal{F}^{\prime}$ than $F^{\prime}$. Then the number of pairs $(G, \mathcal{C})$ with $G \in \mathcal{F}^{\prime} \cap \mathcal{C}$ and $\mathcal{C} \in \mathbf{C}_{F, F^{\prime}}$, is at most $m_{s}\left|F^{\prime} \backslash F\right| ! \alpha_{F} \beta_{F^{\prime}}=m_{s} \mathbf{C}_{F, F^{\prime}}$. Summing over all pairs $F, F^{\prime}$ with $b\left(F, F^{\prime}\right)<s$, we obtain that the umber of pairs $(G, \mathcal{C})$ with $G \in \mathcal{F}^{\prime} \cap \mathcal{C}$ and $\mathcal{C} \in \mathbf{C}_{<s}$, is at most $m_{s} \mathbf{C}_{<s} \leqslant m_{s} n$ !.

As a consequence, the number of pairs $(F, \mathcal{C})$ with $F \in \mathcal{F}^{\prime} \cap \mathcal{C}$ and $\mathcal{C} \in \mathbf{C}_{\geqslant s}$ is at least $\frac{\varepsilon}{2} n$ !. Observe that $\left|\mathbf{C}_{F, F^{\prime}}\right| \leqslant|F| !\left(\left|F^{\prime}\right|-|F|\right) !\left(n-\mid F^{\prime}\right)$ ! and thus the number of pairs $\left(F^{\prime \prime}, \mathcal{C}\right)$ with $F^{\prime \prime} \in \mathcal{F}^{\prime} \cap \mathcal{C}$ and $\mathcal{C} \in \mathbf{C}_{F, F^{\prime}}$ is at most $\left(\left|F^{\prime}\right|-|F|+1\right)|F| !\left(\left|F^{\prime}\right|-|F|\right) !\left(n-\mid F^{\prime}\right) !$. Also if $b\left(F, F^{\prime}\right) \geqslant s$, then $\left|F^{\prime}\right|-|F| \geqslant m_{s}$. Fixing $|F|$ and using $\ell \leqslant|F|,\left|F^{\prime}\right| \leqslant n-\ell$, it is easy to see that $\left(\left|F^{\prime}\right|-|F|+1\right)|F| !\left(\left|F^{\prime}\right|-|F|\right) !\left(n-\left|F^{\prime}\right|\right)$ ! is convex in $\left|F^{\prime}\right|$ and thus it is maximized either at $\left|F^{\prime}\right|-|F|=m_{s}$ or when $\left|F^{\prime}\right|=n-\ell$. Plugging in one obtains that the maximum is taken when $\left|F^{\prime}\right|-|F|=m_{s}$. Using 2. from above, we obtain that for one fixed $\mathbf{C}_{F, F^{\prime}} \subseteq \mathbf{C}_{\geqslant s}$ the number of pairs $\left(F^{\prime \prime}, \mathcal{C}\right)$ with $F^{\prime \prime} \in \mathcal{F}^{\prime} \cap \mathcal{C}$ and $\mathcal{C} \in \mathbf{C}_{F, F^{\prime}}$ is at most

$$
\begin{aligned}
\left(m_{s}+1\right) m_{s} !|F| !\left(n-|F|-m_{s}\right) ! & \leqslant\left(m_{s}+1\right) m_{s} ! \frac{4^{m_{s}}}{n^{m_{s}}}|F| !(n-|F|) ! \\
& \leqslant\left(m_{s}+1\right) m_{s} ! \frac{4^{m_{s}} 10}{\varepsilon n^{m_{s}-0.5}}\lfloor n / 2\rfloor !\lceil n / 2\rceil !
\end{aligned}
$$


Therefore the number of pairs $F, F^{\prime}$ with $b\left(F, F^{\prime}\right) \geqslant s$ and thus the number of copies of $D_{s}$ in $\mathcal{F}^{\prime}$ is at least

$$
\frac{\varepsilon^{2}}{20\left(m_{s}+1\right) ! 4^{m_{s}}} n^{m_{s}-0.5}\left(\begin{array}{c}
n \\
\lfloor n / 2\rfloor
\end{array}\right)
$$

as claimed.

The proofs of (ii) and (iii) are basically the same. Instead of $b\left(F, F^{\prime}\right)$ one introduces $a\left(F, F^{\prime}\right)$, which is the maximum size of an antichain in $\mathcal{F}^{\prime} \cap\left[F, F^{\prime}\right]$, and partitions $\mathbf{C}_{n}$ into $\mathbf{C}_{<s}$ and $\mathbf{C}_{\geqslant s}$ according to $a\left(F, F^{\prime}\right)$. If $a\left(F, F^{\prime}\right)<s$, then $\mathcal{F}^{\prime} \cap\left[F, F^{\prime}\right]$ is induced $D_{s}$-free, so for (ii), instead of Lemma 9, one applies Lemma 10 (i), and for (iii), one applies Lemma 10 (ii) to $\mathbf{C}_{<s}$. The number of induced copies of $D_{s}$ is at least the number of pairs with $a\left(F, F^{\prime}\right) \geqslant s$ and the computation to obtain a lower bound for this number is the same as in (i).

\section{Acknowledgements}

We are very grateful to the referee for the careful reading of the paper and for his/her detailed comments which helped us to improve the manuscript considerably.

Research partially sponsored by the National Research, Development and Innovation Office - NKFIH under the grants K 116769, K 132696, KH 130371, SNN 129364, FK 132060, and KKP-133819. Research of Vizer was supported by the János Bolyai Research Fellowship of the Hungarian Academy of Sciences and by the New National Excellence Program under the grant number ÚNKP-20-5-BME-45. Patkós acknowledges the financial support from the Ministry of Education and Science of the Russian Federation in the framework of MegaGrant no 075-15-2019-1926.

\section{References}

[1] J. Balogh, R. Mycroft and A. Treglown. A random version of Sperner's theorem. Journal of Combinatorial Theory, Series A, 128:104-110, 2014.

[2] J. Balogh and A. Zs. Wagner. Kleitman's conjecture about families of given size minimizing the number of $k$-chains. Advances in Mathematics, 330:229-252, 2018.

[3] B. Bukh. Set Families with a Forbidden Subposet. The Electronic Journal of Combinatorics, 16(1):\#R142, 2009.

[4] T. Carroll and Gy. O. H. Katona. Bounds on maximal families of sets not containing three sets with $A \cap B \subseteq C, A \nsubseteq B$. Order, 25(3):229-236, 2008.

[5] S. Das, W. Gan and B. Sudakov. Sperner's theorem and a problem of Erdős, Katona and Kleitman. Combinatorics, Probability and Computing, 24(4):585-608, 2015.

[6] A. P. Dove, J. R. Griggs, R. Kang and J. S. Sereni. Supersaturation in the Boolean lattice. Integers: Electronic Journal of Combinatorial Number Theory, 4:1-7, 2014.

[7] P. Erdős. On a lemma of Littlewood and Offord. Bulletin of the American Mathematical Society, 51(12):898-902, 1945. 
[8] D. Gerbner and B. Patkós. Extremal finite set theory. CRC Press, 2018.

[9] J. R. Griggs and W-T. Li. The partition method for poset-free families. Journal of Combinatorial Optimization, 25(4):587-596, 2013.

[10] J. R. Griggs, W-T. Li and L. Lu. Diamond-free families. Journal of Combinatorial Theory, Series A, 119(2):310-322, 2012.

[11] J. R. Griggs and L. Lu. On families of subsets with a forbidden subposet. Combinatorics, Probability and Computing, 18(5):731-748, 2009.

[12] K. A. Hogenson. Random and deterministic versions of extremal poset problems. PhD thesis, Iowa State University, 2016.

[13] Gy. O. H. Katona and T. Gy. Tarján. Extremal problems with excluded subgraphs in the $n$-cube. In: Graph Theory, 84-93, 1983.

[14] D. Kleitman. A conjecture of Erdős-Katona on commensurable pairs among subsets of an $n$-set. In: Theory of Graphs, Proceedings of Colloquium held at Tihany, Hungary, 187-207, 1966.

[15] D. Kleitman. On Dedekind's problem: the number of monotone Boolean functions. Proceedings of the American Mathematical Society, 21(3):677-682, 1969.

[16] A. D. Korshunov. The number of monotone Boolean functions (in Russian). Problemy Kibernetiki, 38:5-108, 1981.

[17] M. C. Neto and R. Morris. Maximum-size antichains in random set-systems. Random Struct. Algorithms, 49(2):308-321, 2016.

[18] B. Patkós. Induced and Non-induced Forbidden Subposet Problems. Electronic Journal of Combinatorics, 22(1):\#P1.30, 2015.

[19] B. Patkós. Supersaturation and stability for forbidden subposet problems. Journal of Combinatorial Theory, Series A, 136:220-237, 2015.

[20] W. Samotij. Subsets of posets minimising the number of chains. Transactions of the American Mathematical Society, 371(10):7259-7274, 2019.

[21] E. Sperner. Ein Satz über Untermengen einer endlichen Menge. Mathematische Zeitschrift, 27(1):544-548, 1928. 